\title{
Genome-wide analysis of WRKY gene family in the sesame genome and identification of the WRKY genes involved in responses to abiotic stresses
}

Donghua $\mathrm{Li}^{1+} \mathbb{B}$, Pan Liu ${ }^{1 \dagger}$, Jingyin Yu${ }^{1}$, Linhai Wang ${ }^{1}$, Komivi Dossa ${ }^{1,2}$, Yanxin Zhang ${ }^{1}$, Rong Zhou ${ }^{1}$, Xin Wei ${ }^{1,3^{*}}$ and Xiurong Zhang ${ }^{1 *}$

\begin{abstract}
Background: Sesame (Sesamum indicum L.) is one of the world's most important oil crops. However, it is susceptible to abiotic stresses in general, and to waterlogging and drought stresses in particular. The molecular mechanisms of abiotic stress tolerance in sesame have not yet been elucidated. The WRKY domain transcription factors play significant roles in plant growth, development, and responses to stresses. However, little is known about the number, location, structure, molecular phylogenetics, and expression of the WRKY genes in sesame.

Results: We performed a comprehensive study of the WRKY gene family in sesame and identified 71 SiWRKYs. In total, 65 of these genes were mapped to 15 linkage groups within the sesame genome. A phylogenetic analysis was performed using a related species (Arabidopsis thaliana) to investigate the evolution of the sesame WRKY genes. Tissue expression profiles of the WRKY genes demonstrated that six SiWRKY genes were highly expressed in all organs, suggesting that these genes may be important for plant growth and organ development in sesame. Analysis of the SiWRKY gene expression patterns revealed that 33 and 26 SiWRKYS respond strongly to waterlogging and drought stresses, respectively. Changes in the expression of 12 SiWRKY genes were observed at different times after the waterlogging and drought treatments had begun, demonstrating that sesame gene expression patterns vary in response to abiotic stresses.

Conclusions: In this study, we analyzed the WRKY family of transcription factors encoded by the sesame genome. Insight was gained into the classification, evolution, and function of the SiWRKY genes, revealing their putative roles in a variety of tissues. Responses to abiotic stresses in different sesame cultivars were also investigated. The results of our study provide a better understanding of the structures and functions of sesame WRKY genes and suggest that manipulating these WRKYS could enhance resistance to waterlogging and drought.
\end{abstract}

Keywords: Drought stress, Expression profiling, Sesame, Waterlogging stress, WRKY

\footnotetext{
*Correspondence: xwei@shnu.edu.cn; zhangxr@oilcrops.cn

${ }^{\dagger}$ Equal contributors

${ }^{1}$ Oil Crops Research Institute of the Chinese Academy of Agricultural Sciences, Key Laboratory of Biology and Genetic Improvement of Oil Crops, Ministry of Agriculture, No.2 Xudong 2nd Road, Wuhan 430062, China Full list of author information is available at the end of the article
} 


\section{Background}

Sesame (Sesamum indicum L.) is an important, and probably the most ancient, oil crop and is grown widely in tropical and subtropical regions of the world [1]. Recently, the demand for sesame has increased, but sesame yields have been poor compared with those of other oil crops (e.g., rapeseed: $1939.3 \mathrm{~kg} / \mathrm{ha}$; soybean: $2498.5 \mathrm{~kg} / \mathrm{ha}$; and peanut: $1657.6 \mathrm{~kg} / \mathrm{ha})$. Average sesame yields were alarmingly low between 2010 and 2014, with only $576.1 \mathrm{~kg} / \mathrm{ha}$ being produced in 73 countries (http://faostat.fao.org). This low yield may be attributable to a variety of factors, although abiotic stresses are certainly one of the most significant.

For sesame, the most important abiotic stresses that limit plant growth, development, and yield are drought and waterlogging. In central China, which is the major sesame production area, sesame is generally planted during the rainy season, when waterlogging is the most significant problem and can decrease sesame yields by more than $80 \%$ [2]. Sesame is also grown extensively in the tropical regions of Africa and South America. Here, drought presents a major challenge and can limit the yield from sesame by affecting the number of capsules produced by each plant [3]. Therefore, there is an urgent requirement to understand the molecular mechanisms that underlie the ability of sesame plants to tolerate both drought and waterlogging stresses.

Abiotic stress responses and gene regulation have been studied in a number of plant species, including Arabidopsis, rice, maize, and tomato. Several families of genes are particularly associated with significant improvements in abiotic stress tolerance, including the WRKY, NAC, and ERF gene families [4-6]. Numerous studies have demonstrated that WRKY genes are expressed strongly and rapidly in response to particular abiotic stresses, including wounding, waterlogging, drought, and salt stress [7-9]. In Arabidopsis, AtWRKY30 is induced by methyl viologen, hydrogen peroxide, arsenic, drought, sodium chloride, and mannitol [10]. Nuruzzaman et al. [11] identified five OsWRKY genes expressed at higher levels in drought-tolerant rice compared with those in drought-susceptible rice under experimental water-deficit conditions. Overexpression of OsWRKY47 increased both the yield and drought tolerance compared with wild-type plants [12]. Meng et al. [13] discovered that 10 selected WRKY genes showed differential expression patterns under waterlogging and drought stress in an apple rootstock. These observations suggest that studying WRKY gene families may provide valuable insights into the mechanism underlying abiotic stress tolerance in plants. Furthermore, although drought and waterlogging may primarily affect plants grown in different parts of the world, very little is known about the identity and functions of WRKY genes in sesame.
Over the last decade, WRKY transcription factors have become one of the most extensively studied gene families involved in regulating plant abiotic stress tolerance [14]. WRKY proteins have one or two unique DNAbinding domains that are approximately 60 amino acids (aa) in length and contain the WRKYGQK sequence followed by a $\mathrm{C}_{2} \mathrm{H}_{2}$ zinc-finger-like motif [15]. The DNA-binding region is designated a WRKY domain because the WRKYGQK aa sequence is completely conserved. The WRKY proteins are classified into three major groups (I-III) based on the number of WRKY domains and the pattern of zinc-finger-like motifs. Group II is further divided into five distinct subgroups (IIa-IIe) [15]. The first identified WRKY gene, SPF1, was cloned from sweet potato (Ipomoea batatas) 20 years ago [16]. Since then, a large number of WRKY genes have been identified, including 74 from Arabidopsis thaliana [17], 103 from Oryza sativa [18], 45 from Hordeum vulgare [19], 119 from Zea mays [20], and many more from other plant species [21-23].

Sequencing of the entire sesame genome, and annotation of its 24,148 putative genes, provides an opportunity to identify all the sesame WRKY genes [24-26]. In this study, 71 WRKY genes were identified from the sesame genome and analyses of their structure, phylogeny, chromosomal distribution and duplication, conserved motifs, and stimulation in response to waterlogging and drought were performed. The results provide insights into the evolution of the sesame WRKYs and their functions in abiotic stress responses. The identification and characterization of these WRKY genes may provide opportunities to improve the stress tolerance of sesame.

\section{Results}

\section{Identification of WRKY family genes in sesame}

All Arabidopsis WRKY protein sequences were used as queries for the Basic Local Alignment Search Tool (BLAST) to identify sesame WRKY proteins. In total, 61 putative WRKY genes were identified and predicted protein sequences without a WRKY domain were excluded. A Hidden Markov Model (HMM) search was also performed against the sesame protein database using the WRKY-domain PF03106. An additional 10 protein sequences containing the complete WRKY domain were identified. In total, 71 WRKY proteins were identified in the sesame genome (Table 1 ).

The 71 sesame WRKY proteins ranged from 122 (SiWRKY6) to 1141 (SiWRKY29) aa in length, with an average length of approximately 390 aa. The molecular weights (MWs) ranged from $14.44 \mathrm{kDa}$ (SiWRKY6) to $125.94 \mathrm{kDa}(\mathrm{Si} W R K Y 29)$. The isoelectric points (pIs) of the WKRY proteins ranged from 4.81 (SiWRKY46 and SiWRKY56) to 9.74 (SiWRKY6), with 39 pIs $<7$ and the remaining pIs $>7$ (Table 1 ). Similar observations were 
Table 1 Informations of SiWRKY genes

\begin{tabular}{|c|c|c|c|c|c|c|}
\hline$\overline{\text { Gene symbol }}$ & Gene locus & Linkage group & Peptide length & $\mathrm{pl}$ & MW & Group \\
\hline SiWRKY1 & SIN_1000785 & scaffold00233 & 186 & 7.26 & 20.85 & NG \\
\hline SiWRKY2 & SIN_1001523 & scaffold00164 & 302 & 5.63 & 33.94 & \|e \\
\hline SiWRKY3 & SIN_1001786 & LG12 & 300 & 6.30 & 33.82 & IIc \\
\hline SiWRKY4 & SIN_1001880 & LG04 & 760 & 6.28 & 84.34 & । \\
\hline SiWRKY5 & SIN_1002759 & scaffold00124 & 355 & 5.95 & 40.01 & III \\
\hline SiWRKYG & SIN_1002960 & scaffold00120 & 122 & 9.74 & 14.44 & \|c \\
\hline SiWRKYY & SIN_1003153 & LG14 & 728 & 5.96 & 78.03 & । \\
\hline SiWRKY8 & SIN_1003599 & scaffold00109 & 367 & No & No & \|c \\
\hline SiWRKY9 & SIN_1003920 & LG05 & 540 & 6.27 & 60.10 & I \\
\hline SiWRKY10 & SIN_1003975 & LG13 & 176 & 9.26 & 20.25 & \|c \\
\hline SiWRKY11 & SIN_1004161 & LG15 & 187 & 9.47 & 21.25 & IIc \\
\hline SiWRKY12 & SIN_1004874 & LG15 & 291 & 9.72 & 31.60 & Ild \\
\hline SiWRKY13 & SIN_1005422 & LG02 & 297 & 9.21 & 32.27 & Ild \\
\hline SiWRKY14 & SIN_1005676 & LG11 & 315 & 4.86 & 34.41 & He \\
\hline SiWRKY15 & SIN_1005706 & LG11 & 201 & 5.13 & 22.75 & III \\
\hline SiWRKY16 & SIN_1006024 & LG08 & 561 & 6.14 & 60.65 & $\mathrm{llb}$ \\
\hline SiWRKY17 & SIN_1006129 & LG07 & 497 & 5.77 & 54.61 & NG \\
\hline SiWRKY18 & SIN_1006550 & LG08 & 513 & 8.28 & 56.11 & I \\
\hline SiWRKY19 & SIN_1006749 & LG12 & 462 & 5.38 & 49.64 & lle \\
\hline SiWRKY2O & SIN_1006978 & LG12 & 573 & 6.44 & 61.81 & $\| \mathrm{lb}$ \\
\hline SiWRKY21 & SIN_1007987 & LG15 & 479 & 6.06 & 52.05 & $\| \mathrm{b}$ \\
\hline SiWRKY22 & SIN_1008040 & LG15 & 511 & 8.04 & 55.40 & I \\
\hline SiWRKY23 & SIN_1009399 & scaffold00057 & 626 & 8.28 & 69.68 & I \\
\hline SiWRKY24 & SIN_1009643 & LG06 & 345 & 9.55 & 38.85 & Ild \\
\hline SiWRKY25 & SIN_1009858 & LG11 & 151 & 6.23 & 17.23 & NG \\
\hline SiWRKY26 & SIN_1010783 & LG01 & 296 & 8.67 & 32.83 & Ila \\
\hline SiWRKY27 & SIN_1010982 & LG11 & 327 & 6.43 & 36.72 & III \\
\hline SiWRKY28 & SIN_1011023 & LG11 & 526 & 5.79 & 58.18 & । \\
\hline SiWRKY29 & SIN_1011192 & LG11 & 1141 & 8.49 & 125.94 & IIC \\
\hline SiWRKY3O & SIN_1011284 & LG11 & 452 & 9.10 & 49.38 & । \\
\hline SiWRKY31 & SIN_1011416 & LG03 & 346 & 9.70 & 37.75 & Ild \\
\hline SiWRKY32 & SIN_1012054 & LG04 & 316 & 5.27 & 35.60 & III \\
\hline SiWRKY33 & SIN_1012055 & LG04 & 337 & 5.70 & 37.18 & III \\
\hline SiWRKY34 & SIN_1012623 & LG06 & 281 & 4.83 & 31.76 & NG \\
\hline SiWRKY35 & SIN_1012631 & LG06 & 293 & 5.26 & 32.48 & IIc \\
\hline SiWRKY36 & SIN_1012891 & LG06 & 336 & 9.70 & 37.73 & Ild \\
\hline SiWRKY37 & SIN_1014111 & LG01 & 332 & 6.31 & 36.70 & lle \\
\hline SiWRKY38 & SIN_1014143 & LG01 & 350 & 5.05 & 39.25 & III \\
\hline SiWRKY39 & SIN_1014268 & LG12 & 723 & 6.13 & 78.07 & । \\
\hline SiWRKY4O & SIN_1014366 & LG12 & 584 & 6.52 & 63.04 & I \\
\hline SiWRKY41 & SIN_1014422 & LG12 & 397 & 7.21 & 43.06 & $\mathrm{llb}$ \\
\hline SiWRKY42 & SIN_1015494 & LG06 & 187 & 8.55 & 21.30 & IIc \\
\hline SiWRKY43 & SIN_1015496 & LG06 & 338 & 5.51 & 37.95 & lle \\
\hline SiWRKY44 & SIN_1016166 & LG03 & 372 & 6.17 & 41.48 & $\| c$ \\
\hline
\end{tabular}


Table 1 Informations of SiWRKY genes (Continued)

\begin{tabular}{|c|c|c|c|c|c|c|}
\hline SiWRKY45 & SIN_1016382 & LG03 & 602 & 5.62 & 64.69 & $\| \mathrm{b}$ \\
\hline SiWRKY46 & SIN_1016491 & LG04 & 365 & 4.81 & 39.49 & Ile \\
\hline SiWRKY47 & SIN_1016829 & LG16 & 591 & 6.08 & 62.98 & Ilb \\
\hline SiWRKY48 & SIN_1017975 & LG01 & 330 & 5.30 & 37.22 & III \\
\hline SiWRKY49 & SIN_1017989 & LG02 & 152 & 9.30 & 17.70 & Ilc \\
\hline SiWRKY50 & SIN_1018215 & LG02 & 564 & 8.06 & 61.82 & 1 \\
\hline SiWRKY51 & SIN_1018227 & LG02 & 334 & 5.59 & 35.28 & $\| c$ \\
\hline SiWRKY52 & SIN_1018859 & LG04 & 316 & 6.00 & 34.30 & $\| c$ \\
\hline SiWRKY53 & SIN_1019334 & LG14 & 316 & 7.74 & 35.63 & $\| c$ \\
\hline SiWRKY54 & SIN_1019555 & LG08 & 444 & No & No & lle \\
\hline SiWRKY55 & SIN_1019627 & LG08 & 312 & 6.11 & 34.81 & $\| \mathrm{c}$ \\
\hline SiWRKY56 & SIN_1019661 & LG08 & 285 & 4.81 & 32.22 & lle \\
\hline SiWRKY57 & SIN_1019937 & LG05 & 504 & 6.16 & 54.40 & $\| \mathrm{b}$ \\
\hline SiWRKY58 & SIN_1020605 & LG06 & 346 & 9.65 & 36.99 & $\| d$ \\
\hline SiWRKY59 & SIN_1020883 & LG06 & 160 & 7.76 & 18.49 & Ilc \\
\hline SiWRKY60 & SIN_1021497 & LG01 & 168 & 6.52 & 18.90 & $\| c$ \\
\hline SiWRKY61 & SIN_1021618 & LG01 & 268 & 8.92 & 29.50 & lla \\
\hline SiWRKY62 & SIN_1021622 & LG01 & 255 & 8.81 & 28.19 & lla \\
\hline SiWRKY63 & SIN_1021665 & LG01 & 334 & 9.54 & 36.05 & Ild \\
\hline SiWRKY64 & SIN_1021953 & LG03 & 308 & 9.30 & 34.42 & lla \\
\hline SiWRKY65 & SIN_1022426 & LG06 & 341 & 6.83 & 38.16 & Ilc \\
\hline SiWRKY66 & SIN_1022971 & LG08 & 493 & 8.37 & 54.34 & $\| \mathrm{b}$ \\
\hline SiWRKY67 & SIN_1023226 & LG06 & 542 & 8.08 & 59.73 & 1 \\
\hline SiWRKY68 & SIN_1026059 & LG10 & 493 & 6.58 & 54.73 & $\| \mathrm{b}$ \\
\hline SiWRKY69 & SIN_1026464 & LG08 & 162 & 8.89 & 18.95 & $\| \mathrm{c}$ \\
\hline SiWRKY7O & SIN_1026809 & LG08 & 365 & 8.85 & 39.90 & $\| \mathrm{b}$ \\
\hline SiWRKY71 & SIN_1026948 & LG08 & 564 & 6.19 & 60.99 & $\| \mathrm{b}$ \\
\hline
\end{tabular}

pl proteins' isoelectric point, $M W$ molecular weight

made in Chinese cabbage [27], which has 56 BcWRKYs with pIs ranging from 4.69 to 10.45 and MWs ranging from $20.44 \mathrm{kDa}$ to $119.84 \mathrm{kDa}$.

\section{Chromosomal locations of and duplication events of the SiWRKY genes}

Of the 71 SiWRKY genes, 65 mapped to 15 sesame linkage groups (LGs), with the exception of LG09. Six genes (SiWRKY1, 2, 5, 6, 8, and 23) mapped to unanchored scaffolds (Fig. 1, Table 1). LG06 contained the greatest number of sesame WRKY genes (10, 15.38\%), whereas LG07, LG10, LG13, and LG16 contained only one gene each.

Syntenic analysis and comparison with the grapevine genome revealed that the sesame genome was duplicated in its entirety approximately 71 million years ago, creating two syntenic subgenomes [26]. Based on the synteny of these subgenomes, we identified 10 pairs of duplicated sesame WRKY genes (Additional file 1, Table 2). We were unable to identify any sesame WRKY genes using datasets for tandemly duplicated genes obtained from PTGBase [28], indicating that the WRKY gene family did not undergo tandem gene duplication; this finding is consistent with a previous report [29]. These results indicate that the WRKY gene family underwent whole genome duplication, without tandem gene duplication events (Table 2).

Classification and phylogenetic analysis of the SiWRKY genes We performed multiple sequence alignments to examine the structural features of each SiWRKY protein (Additional file 2). The results showed that 69 SiWRKY proteins contained one or two identical WRKYGQK domains. Although the WRKYGQK domain is highly conserved in WRKY proteins, SiWRKY59 and SiWRKY60 differed at one residue, with a glutamine being replaced by a lysine residue; this change is also found in WRKYs from tomato, Arabidopsis, and other plant species [13, 15, 19, 22]. Additionally, most of the 


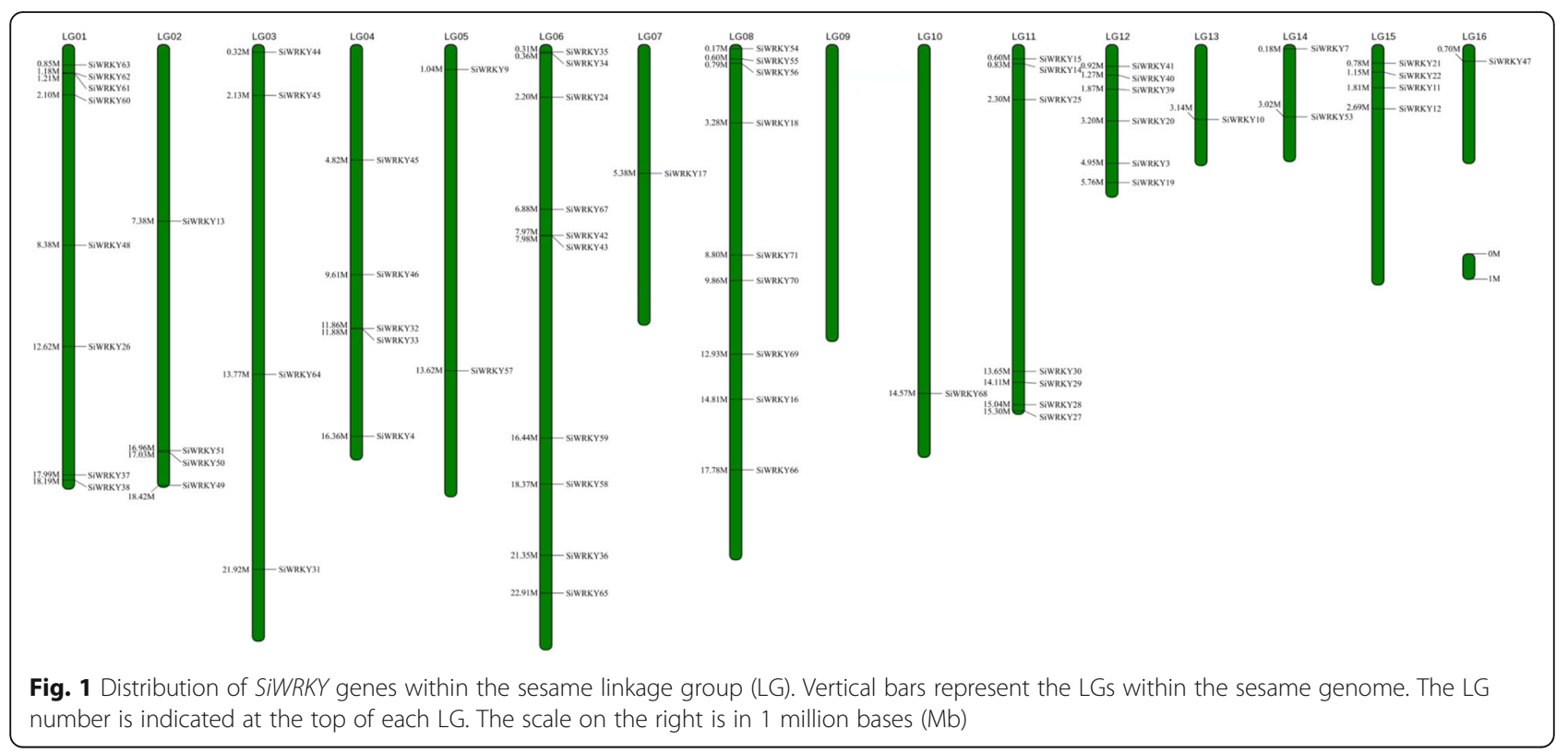

SiWRKY proteins contained the $\mathrm{C}-\mathrm{X}_{4-7}-\mathrm{C}-\mathrm{X}_{23}-\mathrm{H}$ motif that forms the $\mathrm{C}_{2} \mathrm{H}_{2} / \mathrm{C}_{2} \mathrm{HC}$-type zinc-finger structure.

A phylogenetic tree was constructed using the neighbor-joining (NJ) method and based on multiple alignments of sesame and Arabidopsis WRKY domain aa sequences [15]. As shown in Fig. 2, the 71 SiWRKYs were classified into three groups (I, II, and III), and the WRKYS in Group II were further subdivided into five subgroups (IIa-e). Groups I, II, and III consisted of 12, 48, and seven SiWRKY proteins, respectively. A total of four, 11, 18, seven, and eight proteins were assigned to subgroups IIa, IIb, IIc, IId, and IIe, respectively.

Conserved motifs and structure of the SiWRKY family genes Using the SiWRKY phylogenetic relationships data, we identified structural features of the sesame WRKYs, including conserved motifs and the locations of exons and introns. Using Multiple Em for Motif Elicitation

Table 2 Genome-wide duplication of SiWRKY genes

\begin{tabular}{lll}
\hline GrapeVine & Subgenome1 & Subgenome2 \\
\hline GSVIVT01008046001 & SiWRKY16 & SiWRKY45 \\
GSVIVT01010525001 & SiWRKY69 & SiWRKY49 \\
GSVIVT01014854001 & SiWRKY7 & SiWRKY39 \\
GSVIVT01021397001 & SiWRKY55 & SiWRKY3 \\
GSVIVT01026965001 & SiWRKY37 & SiWRKY14 \\
GSVIVT01027069001 & SiWRKY38 & SiWRKY15 \\
GSVIVT01030258001 & SiWRKY28 & SiWRKY9 \\
GSVIVT01033063001 & SiWRKY11 & SiWRKY10 \\
GSVIVT01033188001 & SiWRKY63 & SiWRKY58 \\
GSVIVT01035426001 & SiWRKY60 & SiWRKY59 \\
\hline
\end{tabular}

(MEME) and InterPro Scan 5, we identified 10 conserved motifs in the sesame WRKYs (Fig. 3, Additional file 3) [30]. Motifs 1 and 4 were annotated as WRKY DNA-binding motifs, which is the fundamental characteristic of WRKY proteins. The motif 4 region sequence is conserved in N-terminal WRKY domains. All SiWRKYs contained at least one of these motifs, indicating the existence of features conserved in the WRKY gene family among the sesame WRKYs identified in this study. Group I proteins had two WRKY domains, each consisting of the conserved aa sequence WRKYGQK and a novel zinc-finger-like motif [15]. Group I might include the original genes from the other groups [30]. The gene structure predictions (Fig. 4) revealed that the SiWRKY genes had between one (SiWRKY14, 15, 10, 11, $49,69,42$, and 6) and 11 (SiWRKY29) introns.

\section{Tissue-specific expression profiling of the SiWRKY genes}

To generate expression profiles of the SiWRKY genes under normal conditions, RNA sequence transcriptome data were collected and analyzed. The expression levels of the 71 SiWRKY genes were obtained on the basis of the reads per kilobase of transcript per million mapped reads (RPKM) values from six tissue samples (roots, stem, flowers, leaves, capsules, and seeds). The RPKM values of the transcripts were clustered hierarchically and displayed in a heat map (Fig. 5).

Quantification of transcript levels expressed in different tissues can be useful in determining gene function. The SiWRKY genes displayed diverse expression patterns, possibly reflecting the distinct roles of the different gene family members. A total of $66.20 \%(47 / 71)$, $30.99 \%$ (22/71), 32.39\% (23/71), 21.13\% (15/71), 43.66\% 


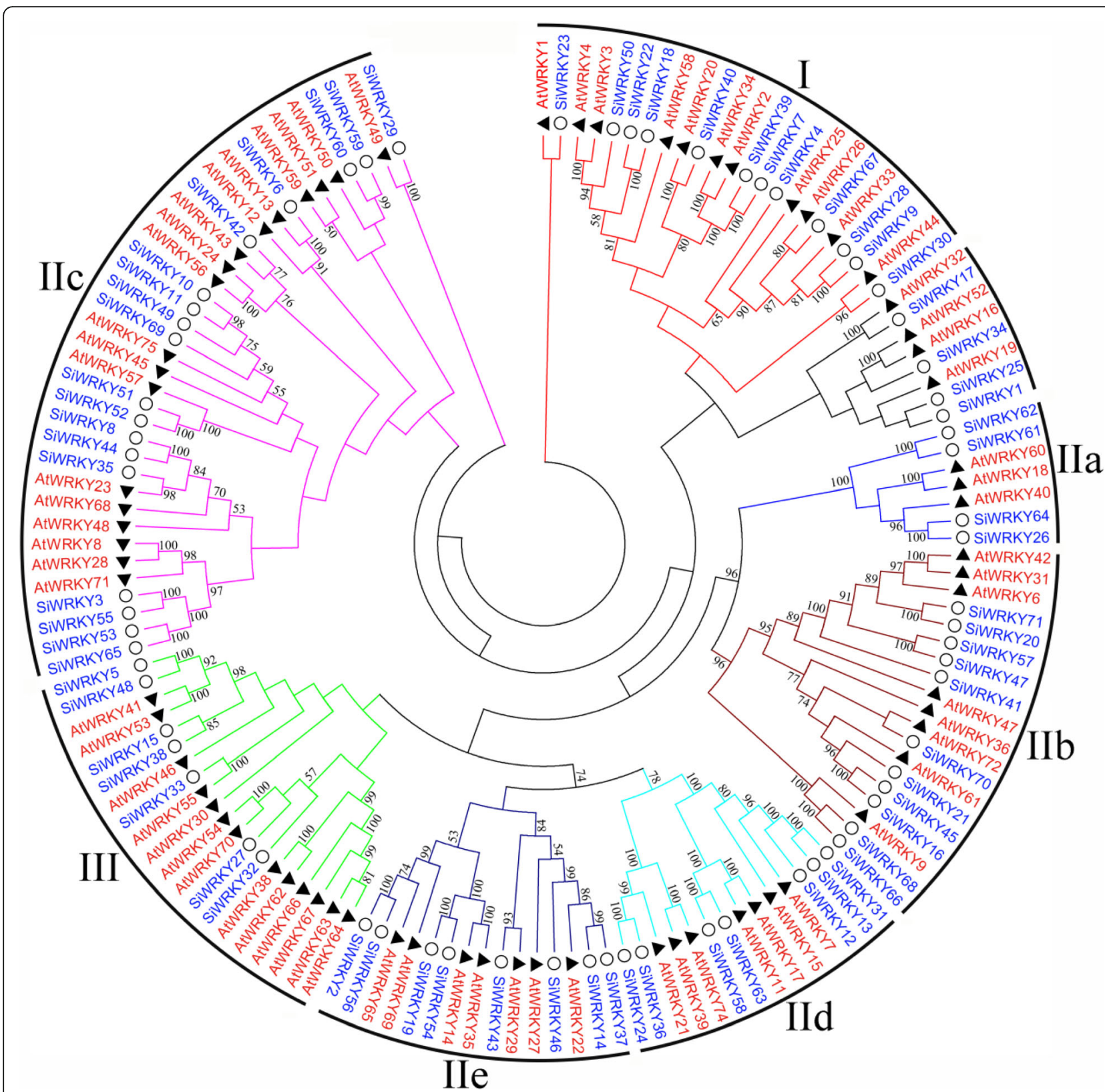

Fig. 2 Phylogenetic analysis of the WRKY proteins in sesame and Arabidopsis. Multiple sequence alignments of WRKY amino-acid sequences were performed using ClustalX, and the phylogenetic tree was constructed using MEGA5 by the neighbor-joining (NJ) method and 1000 bootstrap replicates. The tree was divided into seven phylogenetic subgroups, designated I, Ila-e, and III. The bootstrap values were $\geq 50 \%$

(31/71), and $21.13 \%(15 / 71)$ of the SiWRKY genes were highly expressed (values $>1$ ) in roots, stem, flowers, leaves, capsules, and seeds, respectively. Most of the SiWRKY genes were expressed in all tissues, although SiWRKY4, SiWRKY32, SiWRKY61, SiWRKY62, and SiWRKY70 were only expressed at low levels. Additionally, six SiWRKY genes (SiWRKY22 and SiWRKY39 in Group I, SiWRKY16 and SiWRKY21 in Group IIb, SiWRKY58 in Group IId, and SiWRKY29 in Group IIc) were continuously expressed at high levels (values $>1$ ) in all six organs, suggesting that these genes may be important for plant growth and organ development.

\section{Expression patterns of SiWRKYs in response to waterlogging and drought stresses}

The expression of WRKY genes has been examined under different stress conditions, including high salinity, drought, and high temperature; however, plant gene expression in response to waterlogging stress has not been studied extensively [13]. In this study, we investigated 


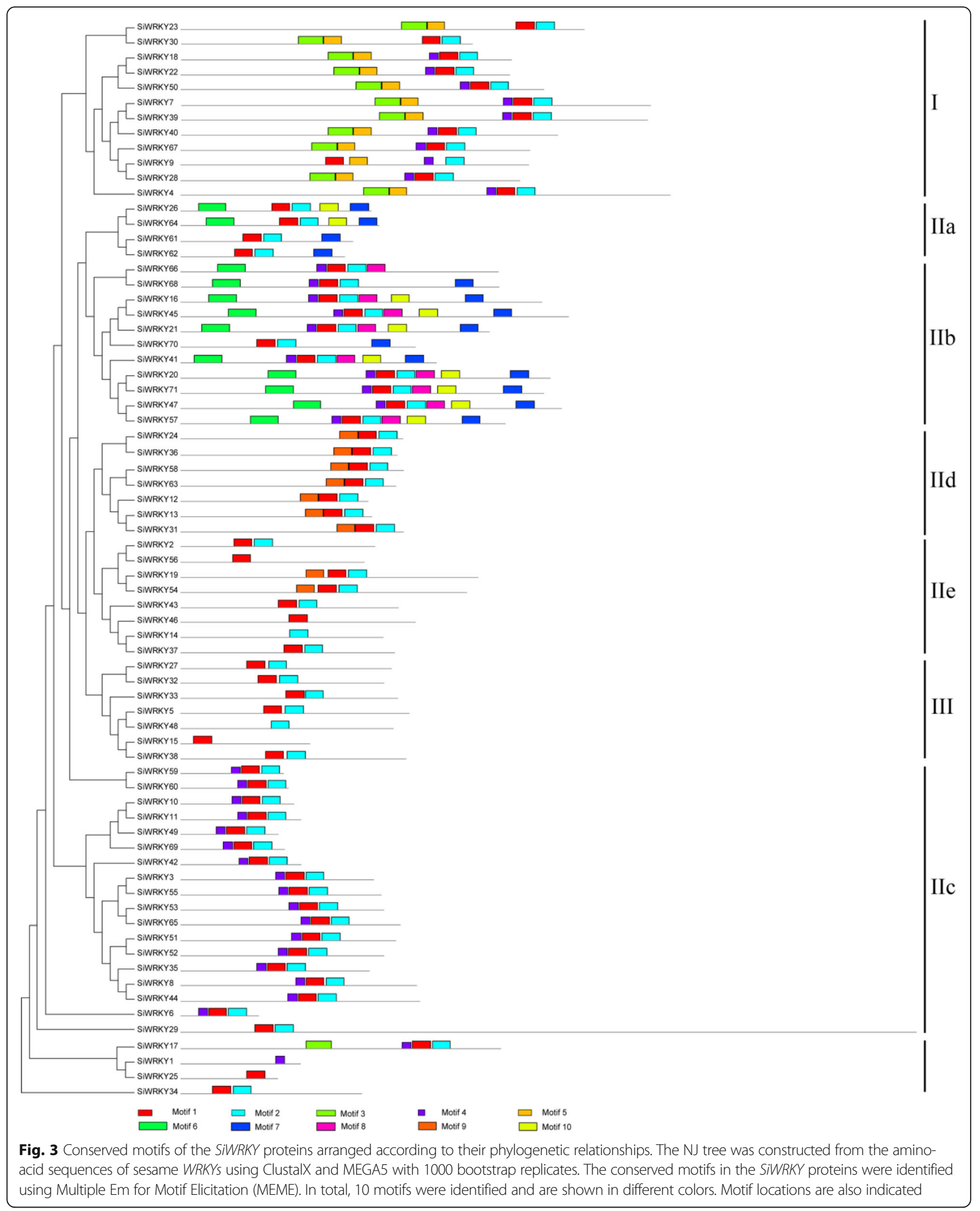

the expression of SiWRKY genes in the roots of sesame cultivars that were tolerant or sensitive to waterlogging stress using quantitative real-time polymerase chain reaction (qRT-PCR). As shown in Fig. 6, the majority of the SiWRKY genes (42 in the tolerant and 40 in the sensitive cultivar) were upregulated in both tolerant and 


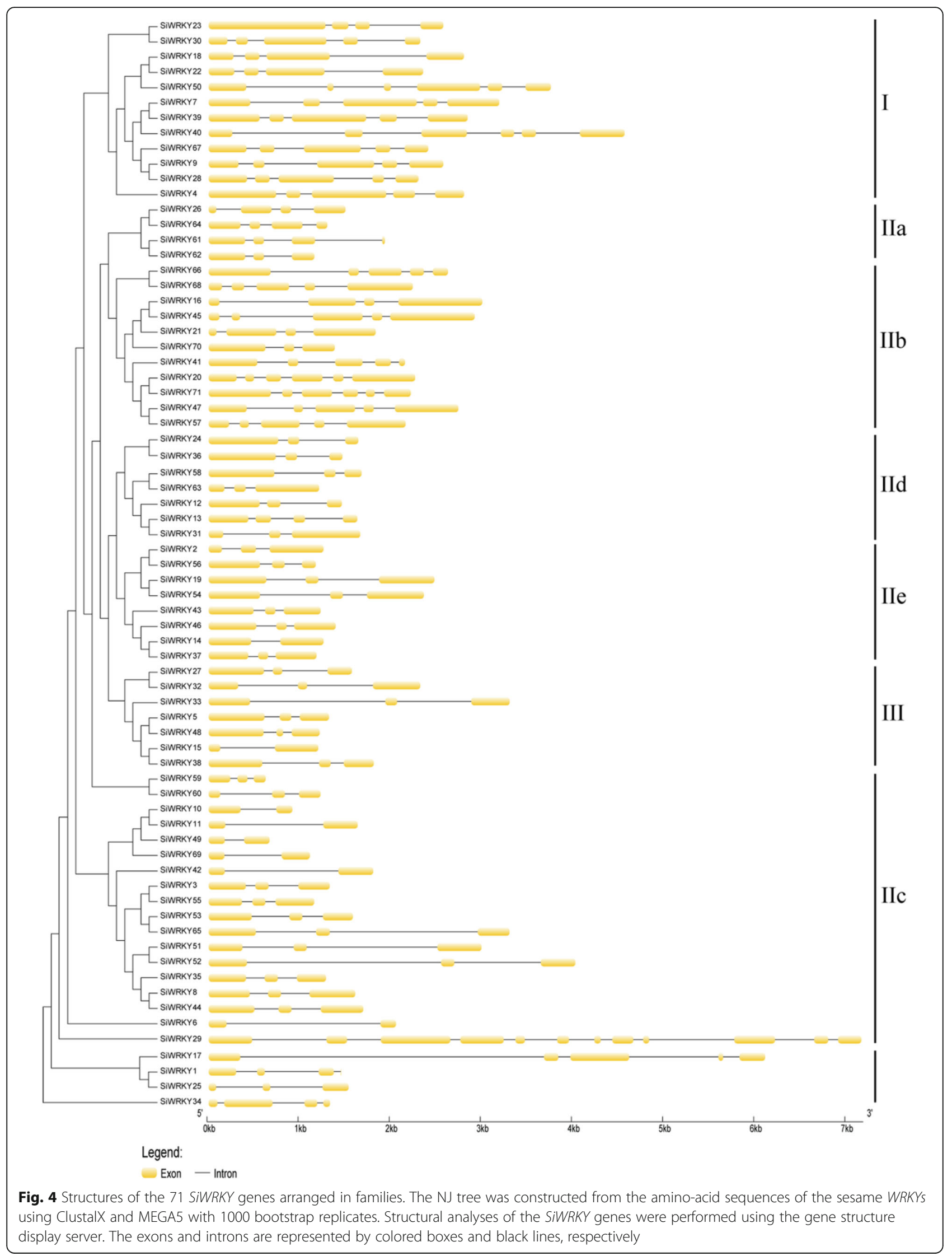




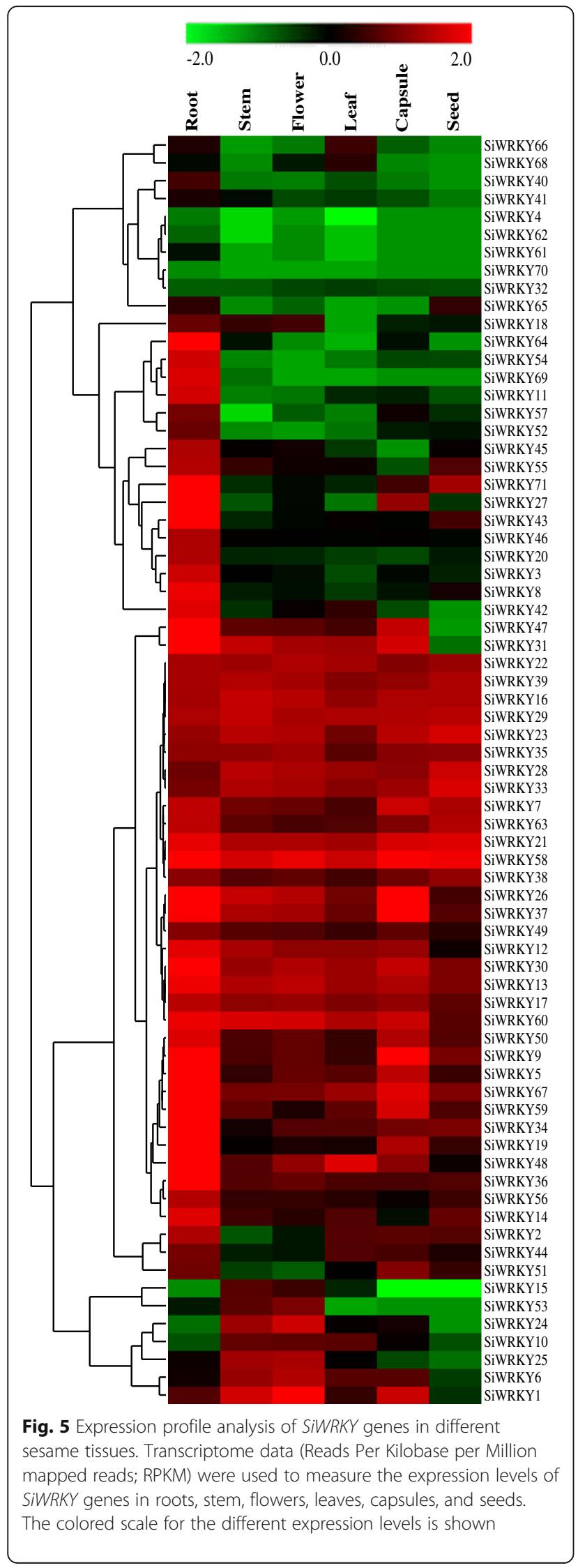

sensitive waterlogged cultivars. Among these upregulated genes, $>2$-fold increases in expression $(P<0.01)$ were observed in 26 of the waterlogging-tolerant cultivars and 22 of the waterlogging-sensitive cultivars. Moreover, the same 18 SiWRKY genes (SiWRKY8, SiWRKY13, SiWRKY16, SiWRKY19, SiWRKY30, SiWRKY35, SiWRKY41, SiWRKY43, SiWRKY46, SiWRKY49, SiWRKY51, SiWRKY54, SiWRKY55, SiWRKY56, SiWRKY64, SiWRKY66, SiWRKY68, and SiWRKY71) displayed $>2$-fold increases in expression level in both waterlogging-tolerant and -sensitive cultivars. In addition, the SiWRKY68 gene exhibited the highest expression level, with $>10$-fold increases in both waterlogging-tolerant and -sensitive cultivars. Waterlogging also decreased the transcript abundance of a large number of SiWRKY genes in roots. In total, 30 (42.3\%) and 29 (40.8\%) SiWRKY genes exhibited $>2$-fold downregulation $(P<0.01)$ in waterlogging-sensitive and -tolerant cultivars, respectively. In particular, the same 15 SiWRKY genes (SiWRKY1, SiWRKY6, SiWRKY7, SiWRKY12, SiWRKY17, SiWRKY27, SiWRKY39, SiWRKY42, SiWRKY47, SiWRKY57, SiWRKY59, SiWRKY60, SiWRKY62, SiWRKY63, and SiWRKY70) displayed exhibited $>2$-fold downregulation in both waterlogging-sensitive and -tolerant cultivars. Therefore, our results show differential expression ( $>2$-fold upregulation or downregulation by a factor of two or more) of 33 genes in both waterlogging-sensitive and -tolerant cultivars, suggesting that these sesame genes play important roles in the response to waterlogging. Additionally, 27 of these 33 genes belong to SiWRKY gene Group II, while only three (SiWRKY7, SiWRKY3O, and SiWRKY39), one (SiWRKY27), and two (SiWRKY1 and SiWRKY17) belong to Group I, Group III, and the unknown group, respectively. Of the 18 upregulated genes, only SiWRKY3O did not belong to Group II. In addition, 29 significant different expression SiWRKYs were found between waterlogging-tolerant and -sensitive cultivars, and 18 of them had high different expression level $(>2$ or $<-2$ ) (Additional file 4).

As shown in Fig. 7, drought stress decreased SiWRKY gene expression in sesame roots. Most of the SiWRKY genes were downregulated in both types of cultivars (53 drought-tolerant and 51 drought-sensitive cultivars). More genes were downregulated by $>2$-fold among the drought-tolerant (32 genes) than in the droughtsensitive (19 genes) cultivars. In contrast, 20 and 18 SiWRKY genes were upregulated in the drought-sensitive and drought-resistant sesame cultivars, respectively. The expression of five genes (SiWRKY11, SiWRKY33, SiWRKY49, SiWRKY55, and SiWRKY59) was upregulated by $>2$-fold and the expression of 19 genes (SiWRKY5, SiWRKY6, SiWRKY8, SiWRKY16, SiWRKY17, SiWRKY21, SiWRKY24, SiWRKY26, 


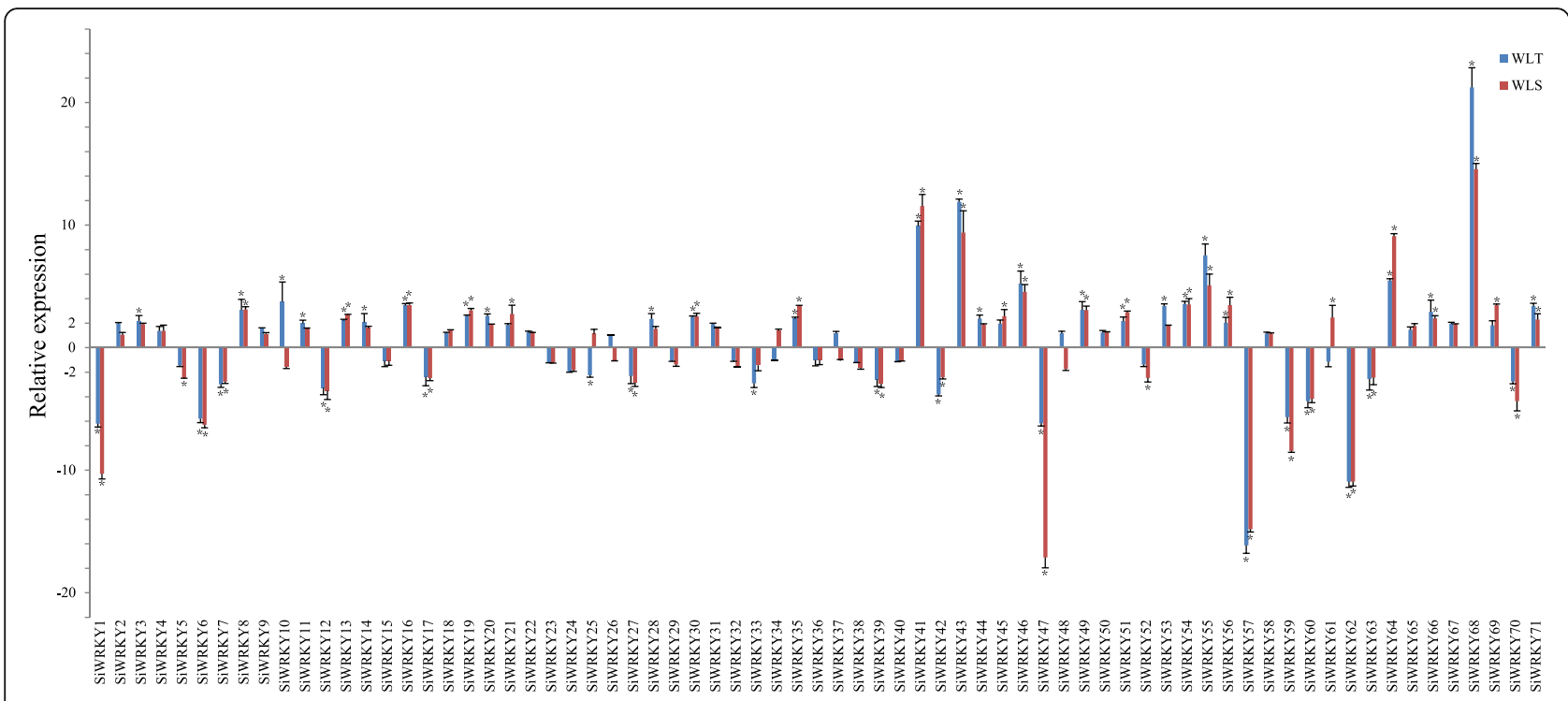

Fig. 6 SiWRKY gene expression in sesame roots treated for 8 h with waterlogging stress compared with untreated controls. Transcript abundance was quantified using quantitative real-time polymerase chain reaction (qRT-PCR) and expression levels were normalized using sesame $\beta$-actin (SIN_1009011) as a reference gene. The mean expression levels from three independent biological replicates were analyzed for significance using t-tests $(p<0.01)$. The histograms represent the relative expression levels and rates of gene induction (stress/control). An asterisk (*) indicates a significant (2-fold) increase in gene expression in plants treated with waterlogging stress compared with untreated controls

SiWRKY27, SiWRKY32, SiWRKY38, SiWRKY40, SiWRKY43, SiWRKY47, SiWRKY48, SiWRKY56, SiWRKY57, SiWRKY62, and SiWRKY70) was downregulated by $>2$-fold. Two SiWRKY genes (SiWRKY42 and SiWRKY61) displayed increases in expression by $>2$-fold in the drought-sensitive cultivar, while decreases in expression by $>2$-fold were detected in the droughttolerant cultivar. These 26 sesame genes displaying marked changes in expression might play important roles in drought stress responses. Eighteen of these genes belonged to Group II, one to Group I, six to Group III, and one to the unknown gene group.

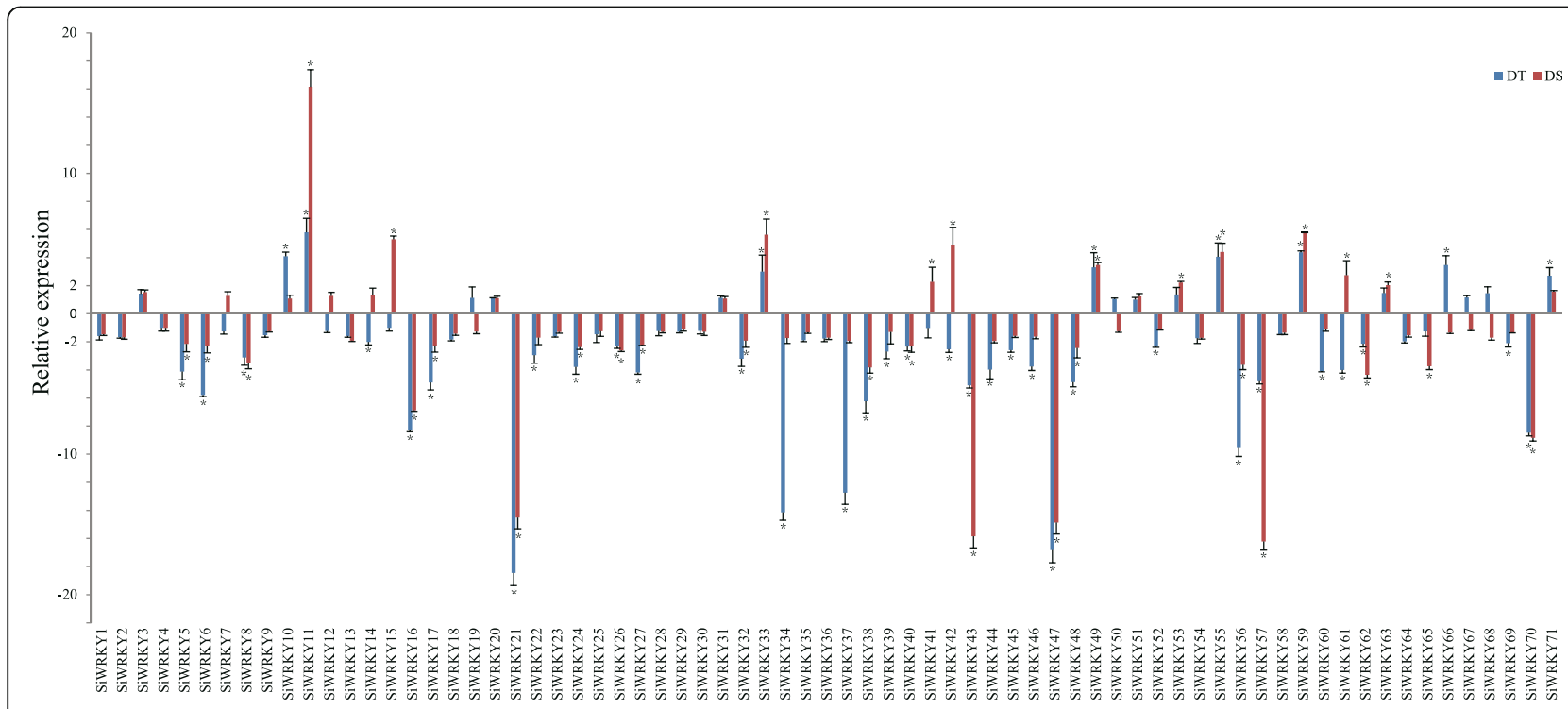

Fig. 7 SiWRKY gene expression in sesame roots treated for $11 \mathrm{~d}$ with drought stress compared with untreated controls. Transcripts abundance was quantified using qRT-PCR and expression levels were normalized using sesame $\beta$-actin (SIN_1009011) as a reference gene. The mean expression levels from three independent biological replicates were analyzed for significance using $t$-tests $(p<0.01)$. The histograms represent the relative expression levels and rates of gene induction (stress/control). An asterisk $\left(^{*}\right)$ indicates a significant (2-fold) increase in gene expression in plants treated with drought stress compared with untreated controls 
Additionaly, 33 significant different expression SiWRKYs were found between drought-tolerant and -sensitive cultivars, and 26 of them had high different expression level $(>2$ or $<-2$ ) (Additional file 5).

\section{Expression of selected SiWRKY genes in response to waterlogging and drought stresses}

To confirm the identities of some of the genes important for waterlogging- and drought tolerance, 12 differential expression SiWRKY genes were selected and their expression levels quantified by qRT-PCR at different timepoints after the onset of each abiotic stress. As shown in
Fig. 8, six SiWRKY genes were expressed at different times following the start of the waterlogging treatment in both the waterlogging-tolerant and -sensitive cultivars $(P<0.05)$. The expression levels of SiWRKY13, SiWRKY35, and SiWRKY43 increased during the waterlogging treatment, although the expression of each gene peaked at a different time. The peak expression of SiWRKY35 occurred before that of SiWRKY13 and SiWRKY43. In contrast, the expression of SiWRKY17, SiWRKY59, and SiWRKY63 was downregulated by waterlogging. For the waterlogging -tolerant and -sensitive cultivars, the difference of the expression of these

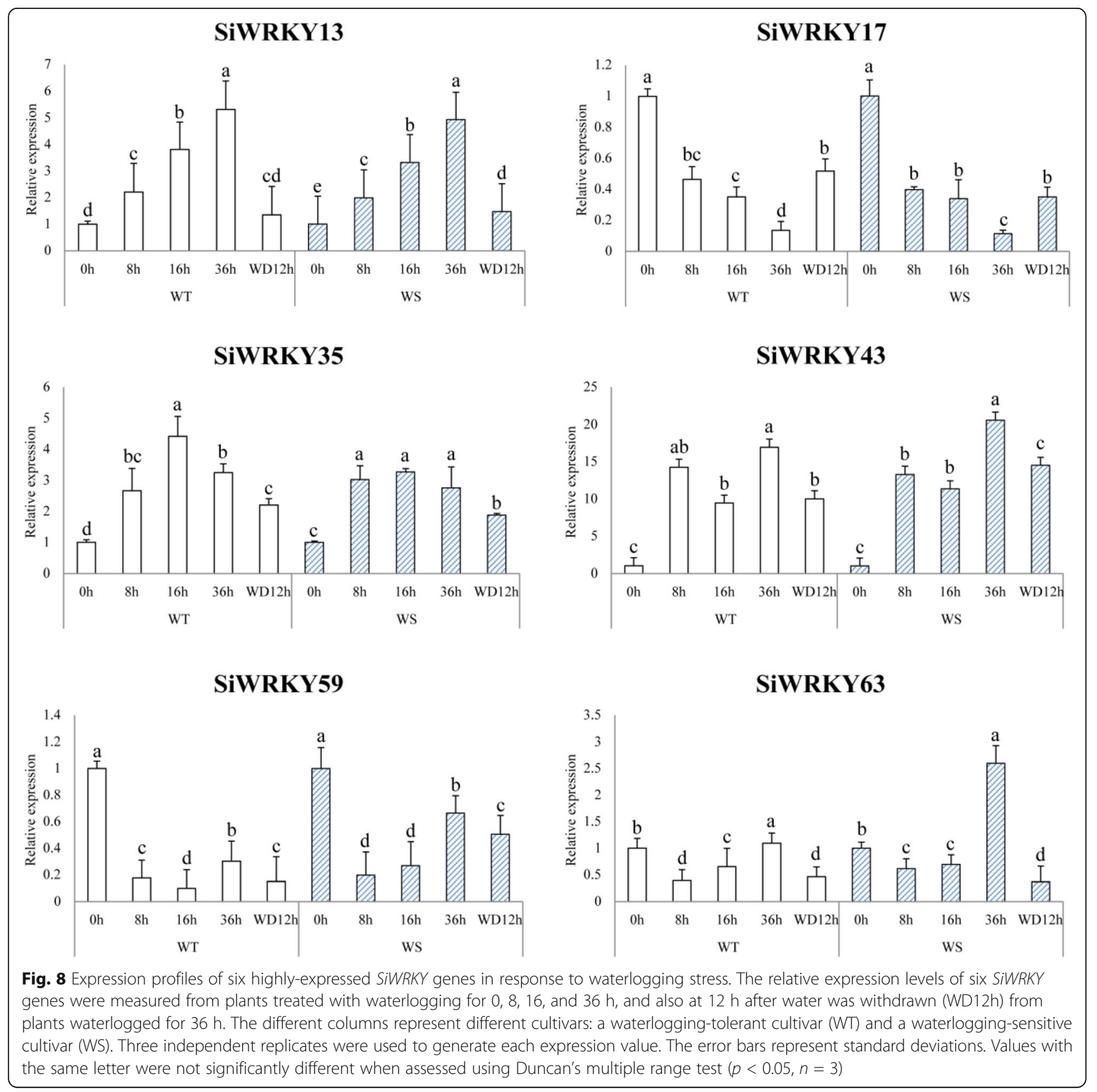


six genes mainly appeared at $36 \mathrm{~h}$. We noticed that SiWRKY35, SiWRKY43, SiWRKY59 and SiWRKY63 expressed in a higher level in waterlogging sensitive cultivar than that in waterlogging tolerant cultivar, especially for the SiWRKY63. This result suggested that the different of the expression of SiWRKY genes might be one of the reasons for the tolerance of waterlogging for sesame varieties.

As shown in Fig. 9, the expression of SiWRKY genes in response to drought differed significantly between drought-tolerant and -sensitive cultivars $(P<0.05)$. The expression of SiWRKY6, SiWRKY11, SiWRKY42, SiWRKY55, and SiWRKY59 was considerably increased by drought stress. However, the expression of SiWRKY6 was suppressed by severe drought conditions (5\% soil water content) and recovered following re-watering (REW), indicating that severe drought decreases the expression of some sesame WRKY genes. Gene expression of SiWRKY under drought stress also showed significant different between drought- tolerant and - sensitive cultivars. Similar to the waterlogging stress, gene expression of most SiWRKY genes in the sensitive cultivar were higher than that in tolerant cultivar. The time-point that the largest difference of the gene expression appeared varied in each gene. For example, SiWRKY11 had a much higher gene expression level in 5\% soil water content of drought-tolerant cultivars, while SiWRKY42 expressed highest in $15 \%$ soil water content.

\section{Discussion}

\section{Number and type of sesame WRKY genes}

The WRKY transcription factor family is one of the most important gene families involved in plant development and stress responses, and WRKY genes have been identified in many species, including Arabidopsis, rice, grape, maize, and cucumber $[15,20,31-33]$. Table 3 summarizes the numbers and types of WRKY genes found in higher plants, and illustrates their diversity among species that have had their genomes sequenced; the number of genes ranges from 55 in cucumber to 343 in rapeseed [34]. In this study, we identified 71 WRKY genes from a total of 27,148 annotated genes in the sesame genome. Relative to the genome size, the sesame WRKY gene family ( $350 \mathrm{Mb}, 71 \mathrm{WRKY}$ genes) is large compared with that of grape, cucumber, and castor bean. However, it is small compared with the WRKY gene families of Arabidopsis (107 Mb, 72 WRKY genes) and rice (440 Mb, 103 WRKY genes). Table 3 shows that one key difference between the sesame, Arabidopsis, and rice genomes is the number of Group III WRKY genes in each. The much more numerous Group III WRKY genes in Arabidopsis and rice is explained by tandem duplication and recent duplication events, which has led to a large-scale expansion of the gene families in these genomes [31, 35]. Recent gene duplication and tandem duplication events are the most important factors in the rapid expansion and evolution of gene families [35]. Previous research has demonstrated that the Arabidopsis Group III WRKY gene family expanded rapidly as a result of recent segmentaland tandem duplication events. Additionally, all of the tandemly duplicated WRKY genes in Arabidopsis belong to Group III, whereas we identified no segmentally or tandemly duplicated Group III WRKY genes in sesame. In this study, we identified 10 pairs of segmentally duplicated SiWRKY genes, but none of these belonged to Group III. Additionally, no SiWRKY genes had been generated by tandem duplication events in the sesame genome. Therefore, the small size of Group III in sesame is probably due to the absence of WRKY gene tandem duplication events.

Conserved motifs and structures of the sesame WRKY genes Almost all of the SiWRKYs contained the WRKYGQK domain, although two Group IIc WRKYs (SiWRKY59 and SiWRKY60) contained the WRKYGKK domain. This variant of the WRKY domain has also been found in pepper [9], tea [36], and apple proteins [13]. Waterlogging induced the expression of SiWRKY59 and SiWRKY60 (Fig. 6), indicating that these genes may be involved in sesame abiotic stress responses.

The conserved motifs and structural features of the sesame WRKYs were identified using MEME and InterPro Scan 5. The InterPro Scan 5 analysis suggested that SiWRKY8 and SiWRKY35 are PWRKY transcription factors, which represented a subset of Group IIc probable WRKY transcription factors from plants. PWRKY transcription factors, with the InterPro number IPR017396, were known to regulate various abiotic stress responses [37]. Thus, SiWRKY8 and SiWRKY35 might regulate the biotic and abiotic stress responses. This is consistent with the high expression of these genes in response to waterlogging. The SiWRKY12, SiWRKY13, SiWRKY24, SiWRKY31, SiWRKY36, SiWRKY58, and SiWRKY63 genes contained Zn-clusterdomain sequences (IPR018872) and encoded WRKYGCM1 zinc-finger-domain proteins, indicating that these genes acquired their functional diversity as developmental and regulatory genes [38]. SiWRKY29 encodes an ATP-dependent metallopeptidase that belongs to the FtsH (IPR005936) protein family [39].

The exon/intron structural diversity found among the SiWRKY genes is related to their evolution [40]. An exon/intron distribution analysis demonstrated that WRKY genes from sesame had greater structural diversity than those from Populus trichocarpa or cassava. Most of the SiWRKY genes (33/71) had two introns, which is common in other plants, including Pyrus 


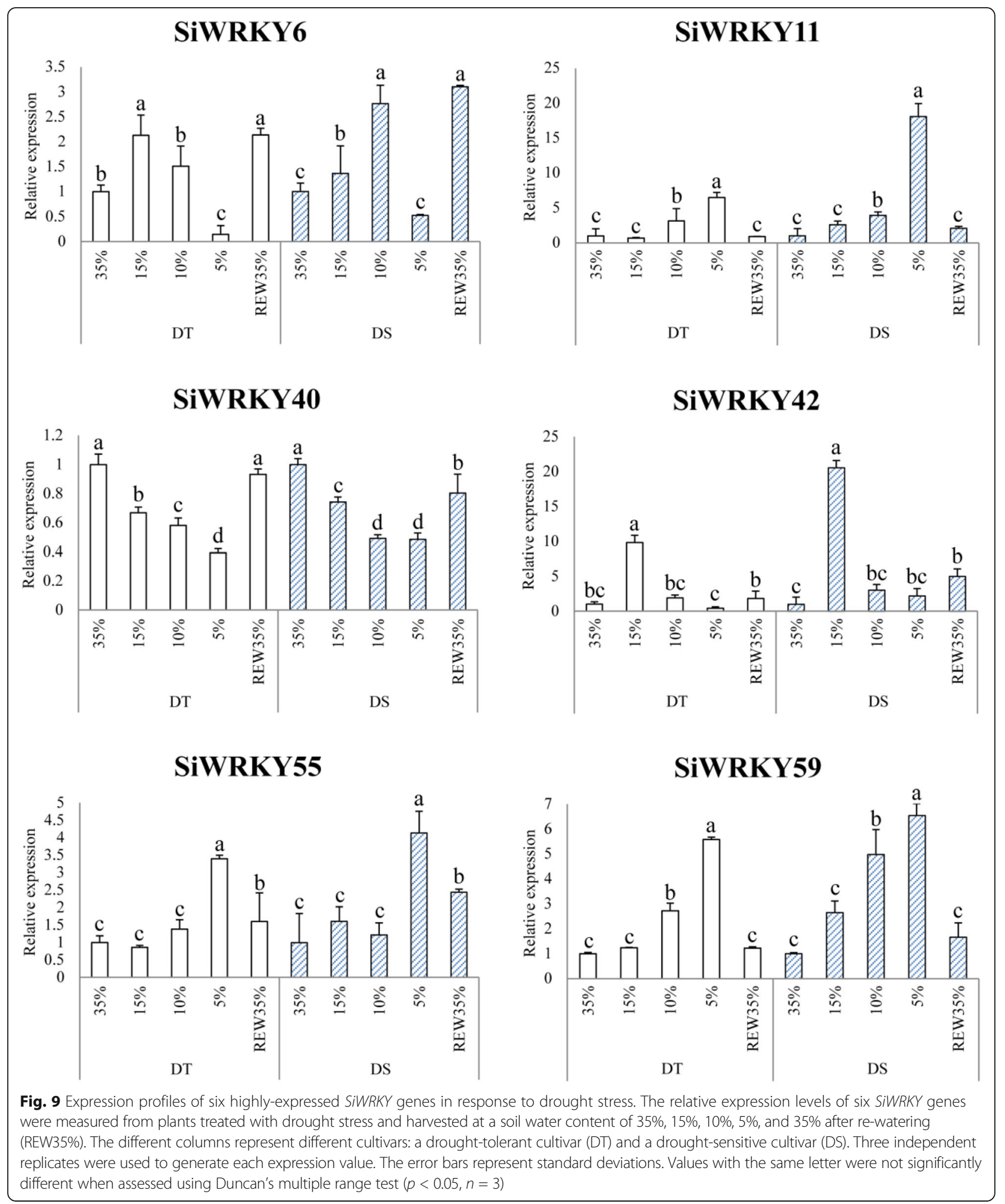

bretschneideri (59/103), Populus trichocarpa (49/104), cassava (42/85), and physic nut $(30 / 58)[4,21,30,41]$. Most of the sesame MADS-box genes $(63.2 \%, 36 / 57)$ have 0 or 1 intron, but most of SiWRKY genes (81.7\%,
58/71) have 2 to 4 introns [25]. The sesame WRKY genes had significantly more introns than the sesame MADS-box genes, indicating that the WRKY gene structure in sesame is more complex. 
Table 3 Numbers and types of WRKY genes in higher plants

\begin{tabular}{|c|c|c|c|c|c|c|c|c|c|c|c|c|}
\hline \multirow[t]{2}{*}{ Plant } & \multirow[t]{2}{*}{ Species } & \multirow[t]{2}{*}{ Genome size } & \multirow[t]{2}{*}{ Name } & \multirow[t]{2}{*}{ Total } & \multicolumn{7}{|c|}{ Group } & \multirow[t]{2}{*}{ NG } \\
\hline & & & & & I & $\| \mathrm{a}$ & $\mathrm{Ilb}$ & Ilc & $\| d$ & Ile & III & \\
\hline sesame & Sesamum indicum & $350 \mathrm{Mb}$ & SiWRKY & 71 & 12 & 4 & 11 & 18 & 7 & 8 & 7 & 4 \\
\hline cucumber & Cucumis sativus & $367 \mathrm{Mb}$ & CsWRKY & 55 & 10 & 4 & 4 & 16 & 8 & 7 & 6 & 0 \\
\hline Arabidopsis & Arabidopsis thaliana & $107 \mathrm{Mb}$ & AtWRKY & 72 & 13 & 4 & 7 & 18 & 7 & 9 & 14 & 0 \\
\hline grape & Vitis vinifera & $490 \mathrm{Mb}$ & VVWRKY & 59 & 12 & 3 & 8 & 15 & 7 & 6 & 6 & 2 \\
\hline rice & Oryza sativa & $440 \mathrm{Mb}$ & OsWRKY & 103 & 15 & 4 & 8 & 15 & 7 & 11 & 36 & 0 \\
\hline tomato & Solanum lycopersicum & $900 \mathrm{Mb}$ & SIWRKY & 78 & 15 & 5 & 8 & 16 & 6 & 17 & 11 & 3 \\
\hline flax & Linum usitatissimum & $373 \mathrm{Mb}$ & LUWRKY & 97 & 24 & 4 & 13 & 16 & 11 & 12 & 15 & 2 \\
\hline soybean & Glycine max & $1.1 \mathrm{~Gb}$ & GmWRKY & 188 & 32 & 14 & 33 & 42 & 21 & 20 & 26 & 0 \\
\hline Castor bean & Ricinus communis & $350 \mathrm{Mb}$ & CbWRKY & 47 & 9 & 3 & 10 & 12 & 3 & 5 & 5 & 0 \\
\hline Brachypodium distachyon & Brachypodium distachyon & $272 \mathrm{Mb}$ & $B d W R K Y$ & 86 & 15 & 3 & 6 & 21 & 6 & 10 & 23 & 2 \\
\hline maize & Zea mays & $2.3 \mathrm{~Gb}$ & ZmWRKY & 136 & 27 & 7 & 11 & 29 & 14 & 17 & 31 & 0 \\
\hline cotton & Gossypium raimondii & $761 \mathrm{Mb}$ & GrWRKY & 116 & 22 & 6 & 16 & 33 & 15 & 12 & 12 & 0 \\
\hline rapeseed & Brassica napus & $630 \mathrm{Mb}$ & $B n W R K Y$ & 343 & 121 & 11 & 34 & 55 & 28 & 30 & 51 & 13 \\
\hline barley & Hordeum vulgare & $5.1 \mathrm{~Gb}$ & HvWRKY & 45 & 8 & 4 & 1 & 11 & 5 & 3 & 13 & 0 \\
\hline pear & Pyrus bretschneideri & $527 \mathrm{Mb}$ & PbWRKY & 103 & 17 & 6 & 10 & 24 & 15 & 16 & 15 & 0 \\
\hline
\end{tabular}

\section{Diverse expression patterns of SiWRKY genes in different} tissues

We analyzed the expression of SiWRKY genes in six different tissues. The results demonstrated variation in the expression patterns of SiWRKY genes. Most SiWRKY genes were highly expressed in roots, whereas a few SiWRKY genes were expressed in developing seeds. This is consistent with observations made in other plants, including rice [18], cucumber [33], grape [42], apple [13], cassava [30], cotton [43], physic nut [41], and cabbage [44]. Our results revealed that SiWRKY genes are expressed tissuespecifically and the high expression levels observed in roots might reflect their roles in responses to abiotic and biotic stresses that first affect plants below ground.

In total, $15 \mathrm{SiWRKY}$ genes were highly expressed in at least five sesame tissues. Six of these genes ( $\mathrm{SiWRKY16,}$ SiWRKY21, SiWRKY22, SiWRKY29, SiWRKY39, and SiWRKY58) were highly expressed in all sesame tissues. Highly expressed WRKY genes usually play important roles in plant development [45]. Therefore, we concluded that the 15 highly expressed SiWRKY genes might be important regulatory factors in sesame development, although further studies are required to verify the function of these genes. Most of these highly expressed SiWRKY genes belong to Groups I and IId. Previous research has demonstrated that Group I WRKY genes are ancestral to other WRKY genes in plants and are more likely to be constitutively expressed in different tissues [46]. For example, the Group I genes $\operatorname{SiWRKY28,}$ SiWRKY29, and SiWRKY67 are expressed in most sesame tissues and highly expressed in response to waterlogging and drought stresses.
In contrast, $17 \mathrm{SiWRKY}$ genes were expressed at low levels in all sesame tissues, and $19 \mathrm{SiWRKY}$ genes were specifically expressed in only one tissue. Among the specifically expressed SiWRKY genes, SiWRKY51 was expressed only in capsules and the remaining $\operatorname{SiWRKY}$ genes were expressed only in roots. These specifically or minimally expressed SiWRKY genes were found in all the WRKY gene subgroups, but many were found in Groups IIc and IIe. A number of Group IIc WRKY genes in Arabidopsis (e.g., AtWRKY8, AtWRKY48, AtWRKY50, and $A t W R K Y 57)$ are involved in responses to bacterial and fungal pathogens, and in the jasmonic acid- and salicylic acid-mediated signaling pathways [27]. Therefore, although some Group IIc SiWRKY genes were expressed at low levels in most sesame tissues, they may play key roles in responses to biotic and abiotic stresses. In this study, SiWRKY51 and SiWRKY65 were highly expressed in the roots of waterlogged plants, whereas SiWRKY10 and SiWRKY53 were highly expressed in response to drought stress. These results indicate that some SiWRKY genes might only be expressed in response to particular abiotic stresses.

\section{Identification of SiWRKY genes involved in responses to abiotic stresses}

Waterlogging and drought are the most serious abiotic stresses for sesame and result in significant losses $(20 \%-50 \%)$ in sesame production within China [24, 47]. However, few abiotic stress tolerance genes have been identified in sesame. Recent research has demonstrated that WRKY genes are involved in responses to various stresses and there is now compelling evidence that 
WRKYs are plant transcription factors that regulate tolerance to abiotic stresses [48]. Gene expression studies have shown that 20 AtWRKY genes in Arabidopsis, 41 OsWRKY genes in rice, 66 GmWRKY genes in soybean, $41 \mathrm{BrWRKY}$ genes in Brassica rapa, and 74 BnWRKY genes in rapeseed are involved in responses to abiotic stresses [14, 18, 34, 49-51]. In this study, 44 SiWRKY genes were expressed differentially in response to waterlogging and drought stresses, indicating that these genes may also be involved in responses to abiotic stresses. To identify the WRKY genes that regulate tolerance to abiotic stresses in sesame, waterlogging- and drought-tolerant and sensitive cultivars were investigated. As shown in Figs. 6 and 7, the expression of some SiWRKY genes differed significantly between the tolerant and sensitive sesame cultivars. For example, SiWRKY10 was highly expressed in tolerant cultivars in response to waterlogging. Further analysis showed that responses to abiotic stresses occurred at different timepoints. SiWRKY17 and SiWRKY59 were highly expressed after $8 \mathrm{~h}$ of waterlogging, whereas the expression of SiWRKY13 and SiWRKY43 peaked at $36 \mathrm{~h}$ after waterlogging began. This suggests that these SiWRKY genes might play important regulatory roles in sesame abiotic stress tolerance and may act at different stages of the stress response.

Compared with the WRKY genes that involved in the response of drought, cold and heat stresses, few WRKY genes that responded to waterlogging stress have been identified in plant. In addition, the expression pattern of WRKY genes under waterlogging stress was also unclear. Therefore, the expression of SiWRKY genes under waterlogging stress and the expression level of six highly expressed SiWRKY genes during the waterlogging treatment were detected in the present study. The qPCR results showed that 33 SiWRKY genes either increase or decrease their expression by a factor of two or more in both waterlogging tolerant and sensitive cultivars. With increasing treatment duration, both up-regulation and down-regulation of the SiWRKY genes were found. Previous studies have revealed that one WRKY gene could function in several disparate signaling pathways. For example, AtWRKY70 functions in plant growth, drought response and cell death [52, 53]. Interestingly, we found that the SiWRKY17 and SiWRKY43 was induced to highly express under waterlogging stress, while the orthologous genes of them, AtWRKY32 and AtWRKY29, was reported to respond to UV irradiation and heat stress, respectively. The result indicated that the orthologous WRKY genes might mediate different pathways and play different roles under the abiotic stress response in different species.

Both phylogeny-based and BLAST-based methods were used to identify WRKY gene orthologs in comparisons of sesame and Arabidopsis. A phylogenetic tree based on WRKY protein sequences from sesame and Arabidopsis was constructed and nodes with bootstrap values $>50$ were used to identify possible orthologs. In addition, standard BLASTP searches were applied to verify possible orthologs of WRKY genes in sesame and Arabidopsis, with relatively strict criteria (Additional file 6). In total, we found 10 orthologous pairs shared between sesame and Arabidopsis (Table 4). The functions of the 10 AtWRKY gene orthologs have been determined and all are involved in responses to abiotic stresses in Arabidopsis. AtWRKY13 and AtWRKY2O regulate tolerance to drought stress $[54,55]$ and their sesame orthologs, SiWRKY6 and SiWRKY40, are also highly expressed in response to drought. Therefore, we conclude that the SiWRKY orthologs of AtWRKY genes also play key roles in the tolerance of abiotic stresses in sesame.

\section{Functional divergence and segmental duplication of WRKY genes}

Recent segmental duplication has occurred frequently in plant genomes because most plants are diploidized polyploids, and many duplicated chromosomal blocks have been retained [28]. Comparisons with grape suggest that the sesame genome underwent a recent genome duplication event, approximately $71 \pm 19$ million years ago [26] In total, 10 pairs of sesame WRKY genes were identified as segmentally duplicated. In the Arabidopsis genome, the Group III WRKY genes are located in a recently segmentally duplicated region of the genome and are highly expressed in response to abiotic stresses. However, in this study, there was little correlation among the expression patterns of the duplicated sesame WRKY genes. For example, SiWRKY16 was highly expressed in all sesame tissues, whereas the duplicated SiWRKY45 gene was only expressed in roots. Additionally, the expression of SiWRKY55 in response to drought was much higher than that of SiWRKY3.

\begin{tabular}{llll}
\multicolumn{4}{l}{ Table 4 Orthologous WRKY genes in sesame and Arabidopsis } \\
\hline Sesame & Arabidopsis & Function & Reference \\
\hline SiWRKY6 & AtWRKY13 & Drought & {$[54]$} \\
SiWRKY17 & AtWRKY32 & UV irradiation, heavy metals & {$[62]$} \\
SiWRKY29 & AtWRKY49 & $\mathrm{H}_{2} \mathrm{O}_{2}$ & {$[63]$} \\
SiWRKY30 & AtWRKY44 & drought & {$[64]$} \\
SiWRKY33 & AtWRKY55 & Oxidative stress & {$[65]$} \\
SiWRKY35 & AtWRKY23 & $\mathrm{H}_{2} \mathrm{O}_{2}$, ABA, mannitol & {$[66]$} \\
SiWRKY40 & AtWRKY20 & Drought & {$[55]$} \\
SiWRKY42 & AtWRKY43 & Nitrogen & {$[67]$} \\
SiWRKY43 & AtWRKY29 & Heat & {$[68]$} \\
SiWRKY67 & AtWRKY26 & Cold & {$[69]$} \\
\hline
\end{tabular}


Research using Arabidopsis, rice, and soybean has focused on identifying the gene targets of WRKYs and understanding the associated regulatory networks. One study showed that co-regulated networks involving WRKY genes were important in regulating the responses of pak-choi to a variety of abiotic stresses [27]. Wheat TaWRKY19 regulates the expression of $D R E B 2 A$, which encodes a key transcription factor that controls the expression of drought-related genes [56]. Therefore, the regulatory roles of WRKY genes in response to abiotic stresses are complex and further studies are required to understand their functions in sesame.

\section{Conclusions}

In this study, we identified a total of 71 sesame WRKY genes and focused on those involved in responses to waterlogging and drought stresses. The distribution, classification, gene structure, and evolutionary characteristics of the sesame WRKY genes were investigated. The differential expression patterns of SiWRKY genes in the tissues of selected cultivars showed that these genes play different roles in sesame development and many exhibit tissue-specific expression patterns. Additionally, SiWRKY gene expression analyses revealed that some were markedly upregulated or downregulated in response to waterlogging and drought stresses. Our results also revealed significant differences in the abiotic-stress-induced expression of WRKYS in stresssensitive and -tolerant sesame cultivars, indicating the involvement of these WRKY genes in abiotic stress tolerance in sesame. In conclusion, our study establishes a structural and functional framework to investigate sesame WRKY proteins. Although the sesame genome was sequenced several years ago, the identification of sesame abiotic-stress-related genes and investigations into their functions are still at an early stage. Our results will facilitate further studies into the functions of WRKY genes important in responses to abiotic stresses and the development of molecular breeding programs to enhance abiotic stress tolerance in sesame.

\section{Methods}

\section{Identification of the WRKY gene family in sesame}

All sesame protein sequences were obtained from the sesame genome database (http://ocri-genomics.org/ Sinbase/) [26]. The Arabidopsis thaliana AtWRKY gene sequences were downloaded from UniProt (http://www.uniprot.org/). The HMM profile for the WRKY DNA-binding domain (PF03106) was downloaded from the PFAM protein families database (http://pfam.xfam.org) and used to identify WRKY genes from the sesame genome with HMMER 3.0 (http://hmmer.janelia.org/). BLAST analyses with all the Arabidopsis WRKYs were used to check the predicted WRKYs from the sesame database. The CDD (http://www.ncbi.nlm.nih.gov/cdd/) and PFAM databases (http://pfam.xfam.org/) were used to validate all the potential sesame WRKY genes identified by HMM and BLAST if they contained a WRKY domain. Multiple sequence alignments were used to confirm the conserved domains from the predicted WRKY sequences.

\section{Chromosomal location and phylogenetic analysis of the WRKY gene family in sesame}

The physical positions of the SiWRKY genes were established using Sinbase (http://ocri-genomics.org/Sinbase/) and mapped to 16 LGs in the sesame genome using MapChart 2.3 [57]. Additionally, Clustal X 2.1 and MEGA 5.2 were used to construct a NJ phylogenetic tree based on the aa sequences of the sesame WRKY domains and selected Arabidopsis WRKYs, with 1000 bootstrap replicates. An alignment of sesame WRKY domains is shown in Additional file 1.

\section{Protein properties and sequence analysis}

Protein MWs and isoelectric points (pIs) were predicted using the ProtParam program (ExPASy tools) based on their deduced aa sequences. The conserved motifs in the full-length WRKY proteins were identified using the MEME program (http://alternate.meme-suite.org/tools/ meme). The parameters employed in the analysis were as follows: maximum number of motifs $=10$; optimum width of motifs $=15-50$ [30]. Additionally, all of the identified motifs were annotated using InterProScan (http://www.ebi.ac.uk/interpro/search/sequence-search).

The exon/intron structures of the SiWRKY genes were determined by comparing their predicted coding sequence (CDS) with genomic sequences using the gene structure display server web-based bioinformatics tool (http://gsds.cbi.pku.edu.cn/) [58].

\section{Analysis of SiWRKY gene expression in different organs using transcriptomic data}

Total RNA was extracted from roots, shoots, leaves, seed capsules, and seeds of Zhongzhi No. 13 grown under normal conditions. RNA pools were constructed using $3 \mu \mathrm{g}$ of RNA per sample according to the manufacturer's instructions and sequenced on a Gene Analyzer II system (Illumina, Inc., San Diego, CA, USA) according to the Illumina RNA-seq protocol. Gene expression levels were calculated in RPKM by taking into account the length of each gene and the read counts mapped. The sesame WRKY gene expression pattern analyses were performed using Gene Cluster 3.0, and the RPKM values for each gene 
in all tissue samples were $\log 10$ transformed. Finally, a heat map was generated using TreeView 1.0.4 [59].

\section{Plant materials and treatments}

Waterlogging-tolerant (WT) cultivar 2541, waterloggingsensitive (WS) cultivar 4508, drought-tolerant (DT) cultivar 0635, and drought-sensitive (DS) cultivar 4728 were all selected from sesame germplasm provided by the Oil Crops Research Institute, Chinese Academy of Agricultural Sciences, Wuhan, China.

For the waterlogging treatment, sesame plants at anthesis were irrigated until the soil surface was covered by a thin layer of water and this was maintained for $36 \mathrm{~h}$. The plants were harvested $8 \mathrm{~h}$ later and their roots were immediately frozen in liquid nitrogen and stored at $-80{ }^{\circ} \mathrm{C}$ prior to further analysis. Control plants were harvested $15 \mathrm{~h}$ before the waterlogging treatment. To further investigate waterlogging resistance-related genes, we harvested plants that had been waterlogged for 0,8 , 16 , and $36 \mathrm{~h}$, and also $12 \mathrm{~h}$ after water was withdrawn (WD12h) from plants waterlogged for $36 \mathrm{~h}$. The roots were harvested as described previously.

For the drought stress treatment, water was withheld for $11 \mathrm{~d}$ from sesame plants at anthesis. Root samples were collected immediately thereafter and frozen in liquid nitrogen prior to analysis [24]. To further investigate genes important for drought resistance, we harvested plants when the soil water content was reached 35\% ( $0 \mathrm{~d}$ controls), 15\% (3 d), 10\% (7 d), 5\% $(11 \mathrm{~d})$, and $35 \%(14 \mathrm{~d})$ after REW. The roots were harvested as described previously.

\section{qRT-PCR analyses of SiWRKY gene expression in response} to waterlogging and drought stresses

RNA was extracted from the roots of each of the four cultivars using the EASYspin Plus Plant RNA Kit (Aidlab Biotechnologies, Beijing, China) [60] according to the manufacturer's instructions. The RNA was quantified using a BIOMATE 3 spectrophotometer (Thermo Scientific, Worcester, MA, USA) and its integrity was confirmed using $1 \%$ agarose gel electrophoresis. A total of $1 \mathrm{mg}$ of RNA was reverse-transcribed into cDNA using the iScript cDNA Synthesis kit (Bio-Rad, Hercules, CA, USA). A control amplicon was generated using the following primers for amplification of $\beta$-actin (SIN_1009011): forward primer, 5'-TTTGAGCAG GAACTGGACACT-3', and reverse primer, 5'-ACAA CACTTCTGGACAACGGA-3'. Gene expression levels were determined by performing qRT-PCR in triplicate on an Icycler iQ5 (Bio-Rad) using the SYBR Green Supermix kit (Bio-Rad), all according to the manufacturer's instructions. Data were analyzed using iQ5 2.1 software (Bio-Rad) and the $2^{-\Delta \Delta C T}$ method [61].

\section{Additional files}

Additional file 1: Synteny of subgenomes in the sesame. The green bars represent the sesame chromosomes. The numbers $01-16$ represent LGs within the sesame genome. Black lines on the green bars indicate the locations of sesame genes within the LGs. Colored lines indicate subgenomes in sesame (PDF $1332 \mathrm{~kb}$ )

Additional file 2: Alignment of SiWRKY domain aa sequences. The alignment was performed using Jalview. The conserved WRKY aa and zinc-finger motifs are highlighted in blue. Gaps are indicated by dashes. (PDF 506 kb)

Additional file 3: Conserved motifs of WRKY proteins in sesame. Significant motifs of more than 10 aa in length were predicted using MEME analysis. The motif IDs, consensus sequence lengths in aa, and e-value of each predicted motif are shown. (PDF $234 \mathrm{~kb}$ )

Additional file 4: The cultivar-specific SiWRKY gene expression in sesame roots treated for $8 \mathrm{~h}$ with waterlogging stress compared with untreated controls in cultivars. Transcript abundance was quantified using quantitative real-time polymerase chain reaction (qRT-PCR) and expression levels were normalized using sesame $\beta$-actin (SIN_1009011) as a reference gene. The mean expression levels from three independent biological replicates were analyzed for significance using $t$-tests $(p<0.01)$. The histograms represent the relative expression levels and rates of gene induction (stress/control). An asterisk $\left(^{*}\right)$ indicates a significant (2-fold) increase in gene expression in plants treated with waterlogging stress compared with untreated controls. (PDF $341 \mathrm{~kb}$ )

Additional file 5: The cultivar-specific SiWRKY gene expression in sesame roots treated for $11 \mathrm{~d}$ with drought stress compared with untreated controls in cultivars. Transcripts abundance was quantified using qRT-PCR and expression levels were normalized using sesame $\beta$-actin (SIN_1009011) as a reference gene. The mean expression levels from three independent biological replicates were analyzed for significance using $t$-tests $(p<0.01)$. The histograms represent the relative expression levels and rates of gene induction (stress/control). An asterisk (*) indicates a significant (2-fold) increase in gene expression in plants treated with drought stress compared with untreated controls. (PDF $544 \mathrm{~kb}$ )

Additional file 6: Synteny between SiWRKY genes in the sesame and Arabidopsis genomes. The green bars represent the chromosomes of the two species. The numbers $01-16$ represent LGs within the sesame genome and the five Arabidopsis chromosomes are labeled Chr1-Chr5. Black lines on the green bars indicate the locations of SiWRKY genes on the chromosomes/within the LGs. Colored lines indicate orthologous genes in sesame and Arabidopsis. (PDF $425 \mathrm{~kb}$ )

\section{Abbreviations}

Aa: amino acid(s); ATP: Adenosine triphosphate; BLAST: Basic Local Alignment Search Tool; BLASTP: BLAST for protein sequences; CDD: Conserved domain database; CDS: Coding DNA sequence; DS: Drought-sensitive; DT: Drought-tolerant; FtsH: Filamentation temperaturesensitive H; GSDS: Gene structure display server; HMM: Hidden Markov model; LGs: Linkage groups; MEME: Multiple Em for Motif Elicitation; MW: Molecular weight; NJ: Neighbor-joining; pl: isoelectric point; qRTPCR: quantitative real-time polymerase chain reaction; REW: After re-watering; RPKM: Reads per kilobase of transcript per million mapped reads;

TD: Tandem duplication; WGD: Whole genome duplication; WS: Waterlogging-sensitive; WT: Waterlogging-tolerant

\section{Acknowledgments}

We sincerely thank Ms. Yuan Gao and Ms. Wenjuan Yang for laboratory assistance and Doc. Jun You for the language editing on the manuscript.

\section{Funding}

This work was funded by the National Natural Science Foundation of China (grant number: 31401412), the China Agriculture Research System (grant number: CARS-14), the Agricultural Science and Technology Innovation Project of the Chinese Academy of Agricultural Sciences (grant number: CAAS-ASTIP2013-OCRI), and by using the Fundamental Research Fund from the Central Non-profit Scientific Institution (grant number: 1610172014003). 


\section{Availability of data and materials}

The datasets supporting the conclusions of this article are included within the article and its additional files; Sesame sequences in this article can be found from the sesame genome database (http://ocri-genomics.org/Sinbase/); The Arabidopsis thaliana gene sequences in this article were downloaded from UniProt (http://www.uniprot.org/). The raw RNA-seq reads and WRKY sequences are available at SesameFG (http://ncgr.ac.cn/SesameFG). All plant materials were selected from sesame germplasm provided by the Oil Crops Research Institute, Chinese Academy of Agricultural Sciences, Wuhan, China.

\section{Authors' contributions}

$\mathrm{DL}, \mathrm{XW}$, and $Z \mathrm{X}$ conceived and designed the experiments. $\mathrm{DL}$ and $\mathrm{PL}$ performed the experiments. DL, PL, XW, KD, JY and RZ analyzed the data. LW and $Y Z$ provided transcriptome data. DL and XW wrote the manuscript. All authors have read and approved the final manuscript.

\section{Ethics approval and consent to participate}

Not applicable.

\section{Consent for publication}

Not applicable.

\section{Competing interests}

The authors declare that they have no competing interest.

\section{Publisher's Note}

Springer Nature remains neutral with regard to jurisdictional claims in published maps and institutional affiliations.

\section{Author details}

Oil Crops Research Institute of the Chinese Academy of Agricultural Sciences, Key Laboratory of Biology and Genetic Improvement of Oil Crops, Ministry of Agriculture, No.2 Xudong 2nd Road, Wuhan 430062, China. ${ }^{2}$ Centre d'Etudes Régional pour I'Amélioration de l'Adaptation à la Sécheresse (CERAAS), BP 3320 Route de Khombole, Thiès, Sénégal. ${ }^{3}$ College of Life and Environmental Sciences, Shanghai Normal University, Shanghai 200234, China.

Received: 23 May 2017 Accepted: 5 September 2017

Published online: 11 September 2017

\section{References}

1. Wei $X$, Zhu X, Yu J, Wang L, Zhang Y, Li D, Zhou R, Zhang X. Identification of sesame genomic variations from genome comparison of landrace and variety. Front Plant Sci. 2016; 10.3389/fpls.2016.01169.

2. Wang L, Zhang Y, Qi X, Li D, Wei W, Zhang X. Global gene expression responses to waterlogging in roots of sesame (Sesamum indicum L.). Acta Physiol Plant. 2012; 10.1007/s11738-012-1024-9.

3. Dossa K, Niang M, Assogbadjo AE, Cissé N, Diouf D, et al. Afr J Biotechnol. 2016; 10.5897/AJB2016.15420

4. Huang X, Li K, Xu X, Yao Z, Jin C, Zhang S. Genome-wide analysis of WRKY transcription factors in white pear (Pyrus bretschneideri) reveals evolution and patterns under drought stress. BMC Genomics. 2015; 10.1186/s12864015-2233-6.

5. Shen J, Lv B, Luo L, He J, Mao C, Xi D, Ming F. The NAC-type transcription factor OsNAC2 regulates ABA-dependent genes and abiotic stress tolerance in rice. Sci Rep. 2017; 10.1038/srep40641.

6. Fan W, Hai M, Guo Y, Ding Z, Tie W, Ding X, Yan Y, Wei Y, Liu Y, Wu C, Shi $\mathrm{H}$, Li K, Hu W. The ERF transcription factor family in cassava: genome-wide characterization and expression analyses against drought stress. Sci Rep. 2016; 10.1038/srep37379.

7. Yoo SJ, Kim SH, Kim MJ, Ryu CM, Kim YC, Cho BH, Yang KY. Involvement of the OsMKK4-OSMPK1 cascade and its downstream transcription factor OsWRKY53 in the wounding response in rice. The Plant Pathology Journal. 2014; 10.5423/PPJ.OA.10.2013.0106.

8. Juntawong P, Sirikhachornkit A, Pimjan R, Sonthirod C, Sangsrakru D, Yoocha T, Tangphatsornruang S, Srinives P. Elucidation of the molecular responses to waterlogging in Jatropha roots by transcriptome profiling. Front Plant Sci. 2014; 10.3389/fpls.2014.00658.
9. Diao WP, Snyder JC, Wang SB, Liu JB, Pan BG, Guo GJ, Wei G. Genome-wide identification and expression analysis of WRKY gene family in Capsicum annuum L. Front Plant Sci. 2016; 10.3389/fpls.2016.00211.

10. Scarpeci TE, Zanor MI, Mueller-Roeber B, Valle EM. Overexpression of AtWRKY30 enhances abiotic stress tolerance during early growth stages in Arabidopsis thaliana. Plant Mol Biol. 2013; 10.1007/s11103-013-0090-8.

11. Nuruzzaman M, Sharoni AM, Satoh K, Kumar A, Leung H, Kikuchi S. Comparative transcriptome profiles of the WRKY gene family under control, hormone-treated, and drought conditions in near-isogenic rice lines reveal differential, tissue specific gene activation. J Plant Physiol. 2014; 10.1016/j. jplph.2013.09.010

12. Raineri J, Wang S, Peleg Z, Blumwald E, Chan RL. The rice transcription factor OsWRKY47 is a positive regulator of the response to water deficit stress. Plant Mol Biol. 2015; 10.1007/s11103-015-0329-7.

13. Meng D, Li Y, Bai Y, Li M, Cheng L. Genome-wide identification and characterization of WRKY transcriptional factor family in apple and analysis of their responses to waterlogging and drought stress. Plant Physiol Biochem. 2016; 10.1016/j.plaphy.2016.02.006

14. Chen L, Song Y, Li S, Zhang L, Zou C, Yu D. The role of WRKY transcription factors in plant abiotic stresses. Biochimica et Biophysica Acta (BBA) - Gene Regulatory Mechanisms. 2012; 10.1016/j.bbagrm.2011.09.002.

15. Eulgem T, Rushton PJ, Robatzek S, Somssich IE. The WRKY superfamily of plant transcription factors. Trends Plant Sci. 2000; 10.1016/S13601385(00)01600-9.

16. Ishiguro S, Nakamura K. Characterization of a CDNA encoding a novel DNAbinding protein, SPF1, that recognizes SP8 sequences in the $5^{\prime}$ upstream regions of genes coding for sporamin and $\beta$-amylase from sweet potato. Mol Gen Genet. 1994; 10.1007/bf00282746.

17. Ülker B, Somssich IE. WRKY transcription factors: from DNA binding towards biological function. Curr Opin Plant Biol. 2004; 10.1016/j.pbi.2004.07.012.

18. Ramamoorthy R, Jiang Sy, Kumar N, Venkatesh PN, Ramachandran S. A comprehensive transcriptional profiling of the WRKY gene family in rice under various abiotic and phytohormone treatments. Plant Cell Physiol. 2008; doi:10.1093/pcp/pcn061.

19. Mangelsen E, Kilian J, Berendzen KW, Kolukisaoglu ÜH, Harter K, Jansson C, Wanke D. Phylogenetic and comparative gene expression analysis of barley (Hordeum vulgare) WRKY transcription factor family reveals putatively retained functions between monocots and dicots. BMC Genomics. 2008; 10. 1186/1471-2164-9-194

20. Wei KF, Chen J, Chen YF, Wu LJ, Xie DX. Molecular phylogenetic and expression analysis of the complete WRKY transcription factor family in maize. DNA Res. 2012; 10.1093/dnares/dsr048.

21. He H, Dong Q, Shao Y, Jiang HY, Zhu S, Cheng B, Xiang Y. Genome-wide survey and characterization of the WRKY gene family in Populus trichocarpa. Plant Cell Rep. 2012; 10.1007/s00299-012-1241-0.

22. Huang S, Gao Y, Liu J, Peng X, Niu X, Fei Z, Cao S, Liu Y. Genome-wide analysis of WRKY transcription factors in Solanum lycopersicum. Mol Gen Genomics. 2012; 10.1007/s00438-012-0696-6.

23. Tripathi P, Rabara RC, Langum TJ, Boken AK, Rushton DL, Boomsma DD, Rinerson Cl, Rabara J, Reese RN, Chen X, Rohila JS, Rushton PJ. The WRKY transcription factor family in Brachypodium distachyon. BMC Genomics. 2012 10.1186/1471-2164-13-270.

24. Dossa K, Wei X, Li D, Fonceka D, Zhang Y, Wang L, Yu J, Boshou L, Diouf D, Cissé N, Zhang $X$. Insight into the AP2/ERF transcription factor superfamily in sesame and expression profiling of DREB subfamily under drought stress. BMC Plant Biol. 2016; 10.1186/s12870-016-0859-4.

25. Wei X, Wang L, Yu J, Zhang Y, Li D, Zhang X. Genome-wide identification and analysis of the MADS-box gene family in sesame. Gene. 2015; 10.1016/j. gene.2015.05.018.

26. Wang L, Yu S, Tong C, Zhao Y, Liu Y, Song C, Zhang Y, Zhang X, Wang Y, Hua W, Li D, Li D, Li F, Yu J, Xu C, Han X, Huang S, Tai S, Wang J, Xu X, Li Y, Liu S, Varshney RK, Wang J, Zhang X. Genome sequencing of the high oil crop sesame provides insight into oil biosynthesis. Genome Biol. 2014; 10. 1186/gb-2014-15-2-r39.

27. Tang J, Wang F, Wang Z, Huang Z, Xiong A, Hou X. Characterization and co-expression analysis of WRKY orthologs involved in responses to multiple abiotic stresses in Pak-choi (Brassica campestris Ssp. chinensis). BMC Plant Biol. 2013; 10.1186/1471-2229-13-188.

28. Yu J, Ke T, Tehrim S, Sun F, Liao B, Hua W. PTGBase: an integrated database to study tandem duplicated genes in plants. Database. 2015; 10.1093/ database/bav017. 
29. Yu J, Wang L, Guo H, Liao B, King G, Zhang X. Genome evolutionary dynamics followed by diversifying selection explains the complexity of the Sesamum indicum genome. BMC Genomics. 2017; 10.1186/s12864-017-3599-4.

30. Wei Y, Shi H, Xia Z, Tie W, Ding Z, Yan Y, Wang W, Hu W, Li K. Genomewide identification and expression analysis of the WRKY gene family in cassava. Front Plant Sci. 2016; 10.3389/fpls.2016.00025.

31. Ross CA, Liu Y, Shen QJ. The WRKY gene family in rice (Oryza sativa). J Integr Plant Biol. 2007; 10.1111/j.1744-7909.2007.00504.x.

32. Zhang $Y$, Feng J. Identification and characterization of the grape WRKY family. Biomed Res Int. 2014; 10.1155/2014/787680.

33. Ling J, Jiang W, Zhang Y, Yu H, Mao Z, Gu X, Huang S, Xie B. Genome-wide analysis of WRKY gene family in Cucumis sativus. BMC Genomics. 2011; 10. 1186/1471-2164-12-471.

34. He Y, Mao S, Gao Y, Zhu L, Wu D, Cui Y, Li J, Qian W. Genome-wide identification and expression analysis of WRKY transcription factors under multiple stresses in Brassica napus. PLoS One. 2016; 10.1371/journal.pone. 0157558.

35. Cannon SB, Mitra A, Baumgarten A, Young ND, May G. The roles of segmental and tandem gene duplication in the evolution of large gene families in Arabidopsis thaliana. BMC Plant Biol. 2004; 10.1186/1471-2229-4-10.

36. Wu Z, Li X, Liu Z, Li H, Wang Y, Zhuang J. Transcriptome-wide identification of Camellia sinensis WRKY transcription factors in response to temperature stress. Mol Gen Genomics. 2016; 10.1007/s00438-015-1107-6.

37. Zhao T, Zhang J, Liang L, Ma Q, Chen X, Zong J, Wang G. Expression and functional analysis of WRKY transcription factors in Chinese wild hazel, Corylus heterophylla Fisch. PLoS One. 2015; 10.1371/journal.pone.0135315.

38. Babu MM, Iyer LM, Balaji S, Aravind L. The natural history of the WRKYGCM1 zinc fingers and the relationship between transcription factors and transposons. Nucleic Acids Res. 2006; 10.1093/nar/gkl888.

39. Janska H, Kwasniak M, Szczepanowska J. Protein quality control in organelles - AAA/FtsH story. Biochim Biophys Acta. 2013; 10.1016/j.bbamcr. 2012.03.016.

40. Shiu S, Bleecker AB. Expansion of the receptor-like kinase/pelle gene family and receptor-like proteins in Arabidopsis. Plant Physiol. 2003; 10.1104/pp.103. 021964.

41. Xiong W, Xu X, Zhang L, Wu P, Chen Y, Li MR, Jiang H, Wu G. Genome-wide analysis of the WRKY gene family in physic nut (Jatropha curcas L.). Gene. 2013; 10.1016/j.gene.2013.04.047.

42. Wang $L$, Zhu W, Fang L, Sun X, Su L, Liang Z, Wang N, Londo JP, Li S, Xin H. Genome-wide identification of WRKY family genes and their response to cold stress in Vitis vinifera. BMC Plant Biol. 2014; 10.1186/1471-2229-14-103.

43. Dou L, Zhang X, Pang C, Song M, Wei H, Fan S, Yu S. Genome-wide analysis of the WRKY gene family in cotton. Mol Gen Genomics. 2014; 10.1007/ s00438-014-0872-y.

44. Tang J, Wang F, Hou X, Wang Z, Huang Z. Genome-wide fractionation and identification of WRKY transcription factors in Chinese cabbage (Brassica rapa Ssp. pekinensis) reveals collinearity and their expression patterns under abiotic and biotic stresses. Plant Mol Biol Report. 2014; 10.1007/s11105-0130672-2.

45. Cheng Y, JalalAhammed G, Yu J, Yao Z, Ruan M, Ye Q, Li Z, Wang R, Feng K, Zhou G, Yang Y, Diao W, Wan H. Putative WRKYs associated with regulation of fruit ripening revealed by detailed expression analysis of the WRKY gene family in pepper. Sci Rep. 2016; 10.1038/srep39000.

46. Zhang $Y$, Wang L. The WRKY transcription factor superfamily: its origin in eukaryotes and expansion in plants. BMC Evol Biol. 2005; 10.1186/14712148-5-1.

47. Wang L, Li D, Zhang Y, Gao Y, Yu J, Wei X, Zhang X. Tolerant and susceptible sesame genotypes reveal waterlogging stress response patterns. PLoS One. 2016; 10.1371/journal.pone.0149912.

48. Banerjee A, Roychoudhury A. WRKY proteins: signaling and regulation of expression during abiotic stress responses. Sci World J. 2015; 10.1155/2015/ 807560.

49. Shankar A, Pandey A, Pandey GK. WRKY transcription factor: role in abiotic and biotic stress. Place: University of Delhi South Campus; 2013.

50. Yu Y, Wang N, Hu R, Xiang F. Genome-wide identification of soybean WRKY transcription factors in response to salt stress. Spring. 2016; 10.1186/s40064016-2647-x.

51. Kayum MA, Jung HJ, Park JI, Ahmed NU, Saha G, Yang TJ, Nou IS. Identification and expression analysis of WRKY family genes under biotic and abiotic stresses in Brassica rapa. Mol Gen Genomics. 2015; 10.1007/ s00438-014-0898-1.
52. Chen J, Nolan TM, Ye H, Zhang M, Tong H, Xin P, Chu J, Chu C, Li Z, Yin Y. Arabidopsis WRKY46, WRKY54, and WRKY70 transcription factors are involved in brassinosteroid-regulated plant growth and drought responses. Plant Cell. 2017; 10.1105/tpc.17.00364.

53. Kaurilind $\mathrm{E}, \mathrm{Xu}$ E, Brosché M. A genetic framework for $\mathrm{H}_{2} \mathrm{O}_{2}$ induced cell death in Arabidopsis thaliana. BMC Genomics. 2015; 10.1186/s12864-015-1964-8.

54. Xiao J, Cheng H, Li X, Xiao J, Xu C, Wang S. Rice WRKY13 regulates cross talk between abiotic and biotic stress signaling pathways by selective binding to different cis-elements. Plant Physiol. 2013; 10.1104/pp.113.226019.

55. Luo X, Bai X, Sun X, Zhu D, Liu B, Ji W, Cai H, Cao L, Wu J, Hu M, Liu X, Tang $L$, Zhu Y. Expression of wild soybean WRKY20 in Arabidopsis enhances drought tolerance and regulates ABA signalling. J Exp Bot. 2013; 10.1093/ jxb/ert073.

56. Niu CF, Wei WEl, Zhou QY, Tian AG, Hao YJ, Zhang WK, Ma B, Lin Q, Zhang ZB, Zhang JS, Chen SY. Wheat WRKY genes TaWRKY2 and TaWRKY19 regulate abiotic stress tolerance in transgenic Arabidopsis plants. Plant Cell Environment. 2012; 10.1111/j.1365-3040.2012.02480.x.

57. Voorrips RE. MapChart: software for the graphical presentation of linkage maps and QTLs. J Hered. 2002; 10.1093/jhered/93.1.77.

58. Hu B, Jin J, Guo AY, Zhang H, Luo J, Gao G. GSDS 2.0: An upgraded gene feature visualization server. Bioinformatics. 2015; 10.1093/bioinformatics/ btu817.

59. de Hoon MJL, Imoto S, Nolan J, Miyano S. Open source clustering software. Bioinformatics. 2004; 10.1093/bioinformatics/bth078.

60. Sun Q, Jiang H, Zhu X, Wang W, He X, Shi Y, Yuan Y, Du X, Cai Y. Analysis of sea-island cotton and upland cotton in response to Verticillium dahliae infection by RNA sequencing. BMC Genomics. 2013; 10.1186/1471-2164-14-852.

61. Livak KJ, Schmittgen TD. Analysis of relative gene expression data using real-time quantitative PCR and the $2^{-\Delta \Delta C T}$ method. Methods. 2001; 10.1006/ meth.2001.1262

62. Mucha S, Walther D, Müller TM, Hincha DK, Glawischnig E. Substantial reprogramming of the Eutrema salsugineum (Thellungiella salsuginea) transcriptome in response to UV and silver nitrate challenge. BMC Plant Biol. 2015; 10.1186/s12870-015-0506-5.

63. Balazadeh S, Jaspert N, Arif M, Mueller-Roeber B, Maurino VG. Expression of ROS-responsive genes and transcription factors after metabolic formation of $\mathrm{H}_{2} \mathrm{O}_{2}$ in chloroplasts. Front Plant Sci. 2012; 10.3389/fpls.2012.00234.

64. Han Y, Zhang X, Wang Y, Ming F. The suppression of WRKY44 by GIGANTEAmiR172 pathway is involved in drought response of Arabidopsis thaliana. PLoS One. 2013; 10.1371/journal.pone.0073541.

65. Tosti N, Pasqualini S, Borgogni A, Ederli L, Falistocco E, Crispi S, Paolocci F. Gene expression profiles of O3-treated Arabidopsis plants. Plant Cell Environment. 2006; 10.1111/j.1365-3040.2006.01542.x.

66. Guo D, Qin G. EXB1/WRKY71 Transcription factor regulates both shoot branching and responses to abiotic stresses. Plant Signal Behav. 2016; 10. 1080/15592324.2016.1150404.

67. Scheible W-R, Morcuende R, Czechowski T, Fritz C, Osuna D, Palacios-Rojas N, Schindelasch D, Thimm O, Udvardi MK, Stitt M. Genome-wide reprogramming of primary and secondary metabolism, protein synthesis, cellular growth processes, and the regulatory infrastructure of Arabidopsis in response to nitrogen. Plant Physiol. 2004; 10.1104/pp.104.047019.

68. Li Y, Williams B, Dickman M. Arabidopsis B-cell lymphoma2 (BCl-2)-associated athanogene 7 (BAG7)-mediated heat tolerance requires translocation, sumoylation and binding to WRKY29. New Phytol. 2017; 10.1111/nph.14388.

69. Soitamo AJ, Piippo M, Allahverdiyeva Y, Battchikova N, Aro E-M. Light has a specific role in modulating Arabidopsis gene expression at low temperature. BMC Plant Biol. 2008; 10.1186/1471-2229-8-13. 